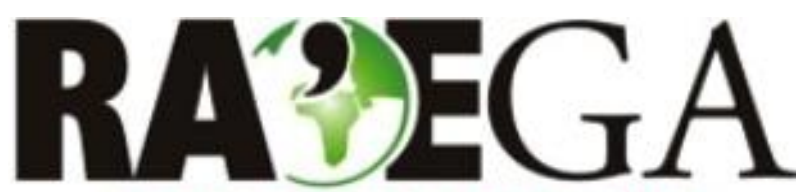

O ESPAÇO GEOGRÁFICO EM ANÁLISE

\title{
ANÁLISE DA INFLUÊNCIA DA ESCALA NA OBTENÇÃO DOS ATRIBUTOS TOPOGRÁFICOS DERIVADOS DE MDE
}

\section{ANALYSIS OF SCALE IN ACQUIRING TOPOGRAPHIC ATTRIBUTES DERIVED FROM MDE}

\author{
Gisele Neuman ${ }^{1}$, Claudinei Taborda da Silveira ${ }^{2}$, Tony Vinicius Moreira Sampaio ${ }^{3}$
}

\section{RESUMO}

O presente trabalho propõe uma análise comparativa entre cinco modelos digitais de elevação (MDEs), na estrutura de grade regular quadrangular, gerados a partir de bases de dados altimétricos de diferentes escalas e, por consequência, com distintas resoluções de grade. Foram utilizados como parâmetros os atributos topográficos derivados do MDE: elevação, declividade, plano e perfil de curvatura. A comparação de dados pontuais foi realizada através de regressão linear, as quais serviram para verificação do relacionamento das variáveis através da representação contínua da superfície topográfica, empregando-se matrizes de confusão (índices de exatidão global e de erros - omissão e inclusão), correspondente às classes representadas na base cartográfica com melhor resolução espacial. Os resultados demonstraram que a redução da resolução espacial do MDE de entrada influencia nos valores dos atributos topográficos, os quais tendem a se concentrar próximos de valores médios. Esse comportamento se apresenta na declividade, em vertentes com inclinações mais suavizadas, e no plano e perfil de curvatura em segmentos planares e retilíneos, respectivamente. É possível afirmar que a resolução dos modelos terá influência direta sobre os parâmetros morfométricos derivados, pois os valores de diferenças advêm da mensuração da variação espacial em função da utilização de matrizes de vizinhança. O refinamento da quantidade de informações interfere nos resultados das aplicações de funções polinomiais tornando as representações um desafio. Isto evidencia a necessidade de utilização de dados em escalas adequadas aos trabalhos a serem realizados, pois os MDEs são importantes dados de análise espacial.

Palavras-chave: declividade; plano de curvatura; perfil de curvatura; modelo digital de elevação

\section{ABSTRACT}

This paper proposes a comparative analysis between five digital elevation models (DEM's), in the form of regular quadrangular grid, generated from altimetric databases at different scales and, as a consequence, with distinct grid resolutions. Topographic attributes derived from the DEM were used as coordinates: elevation, slope, plane and curvature profile. A comparison of point data was performed through linear regression as used to verify the price of variables through the continuous representation of the topographic surface, using confusion matrices (indices of global accuracy and errors - omission and inclusion), corresponding as classes represented in the cartographic base with better spatial resolution. The results demonstrates that reducing the spatial resolution of the DEM input influences substantially the value of topographical attributes, which tend to concentrate near the mean values. This behavior is present in the slope with smoother gradients, and plan and profile curvature shows planar and rectilinear segments, respectively. It is possible that the solution of the models has a direct influence on the morphometric parameters, the advance values of the measurement of the spatial variation in the function of the use of neighborhood matrices. The refinement of the amount of information interferes in the results of applying polynomial functions making representations a challenge. This evidences the need to use data at scales appropriate to the work to be performed, since the spatial analysis tools of the MDE are important.

Key-words: plane curvature; profile curvature; digital elevation model.

Recebido em: 10/11/2017

Aceito em: 09/02/2018

\footnotetext{
${ }^{1}$ Universidade Federal do Paraná, Curitiba/PR, e-mail: giseleneuman@gmail.com

2 Universidade Federal do Paraná, Curitiba/PR, e-mail: claudineits@ufpr.br

${ }^{3}$ Universidade Federal do Paraná, Curitiba/PR, e-mail: tonysampaio@ufpr.br
} 


\section{ANÁLISE DA INFLUÊNCIA DA ESCALA NA OBTENÇÃO DOS ATRIBUTOS TOPOGRÁFICOS DERIVADOS DE MDE}

\section{INTRODUÇÃO}

$O$ aumento da disponibilidade de modelos digitais de representação da superfície terrestre traz como principal consequência uma mudança de paradigmas com relação à geomorfometria. A utilização das soluções advindas das análises morfométricas do terreno possibilitam a implementação de novas soluções para uma análise multiescalar e deteç̧ão de características do relevo em diversas escalas, na definição da direção de escoamento de fluxo hídrico (BUARQUE et al., 2009), para o mapeamento de solos apoiando-se nas formas do relevo (CHAGAS, 2006; SILVEIRA et al.,2012c; SILVEIRA et al.,2013a; NOWATZKI, 2013), apoio à cartografia geomorfológica (SILVEIRA et al., 2014a; SAMPAIO E AUGUSTIN, 2014), análises morfotectônicas apoiado em anomalias na morfologia (SALAMUNI et al., 2008), análise do fator de segurança da estabilidade de vertentes (SILVEIRA et al.,2012a), identificação de áreas susceptíveis a ocorrência de escorregamentos de encosta (GUIMARÃES et al. 2003; GOMES et al. 2013), avaliação de suscetibilidade ambiental considerando o relevo como condicionante (PEREIRA e PEREIRA, 2011; SILVEIRA et al.,2013b; SILVEIRA et al.,2014b), dentre outras aplicações.

$\mathrm{Na}$ maior parte dos casos a geomorfometria emprega modelos digitais estruturados em grades, sendo as mais utilizadas as triangulares e as regulares, compostas por matrizes com valores de elevação da superfície do terreno. A estrutura de grade permite a realização de cálculos de funções polinomiais entre o valor local e de sua vizinhança possibilitando a extração de informações que são denominadas de atributos topográficos (MOORE et al., 1993).

Os atributos topográficos representam mensurações quantitativas da superfície terrestre. Aparecem na literatura com outras distintas designações, dentre elas, variáveis morfométricas (SHARY et al., 2002), variáveis geomorfométricas (BISPO et al., 2009), atributos do terreno (PENNOCK, 2003; CÁTEN, 2009), atributos geomorfométricos (SCHMIDT e DIKAU,
1999) e, atributos do relevo (IPPOLITI et al., 2005; SIRTOLI et al., 2008).

Os modelos digitais de elevação (MDE) são importantes fontes de informação em países como o Brasil, pois mesmo com a grande quantidade de informações produzidas sobre o relevo, estas não possuem o detalhamento necessário em algumas regiões e é praticamente inexistente em outras.

Esses modelos são essenciais para a geração de dados para o planejamento pois podem ser facilmente obtidos a partir de processos fotogramétricos, cartas topográficas e sensores diversos.

Atualmente o modelo mais utilizados no Brasil é o Shuttle Radar Topographic Mission (SRTM), com resolução espacial aproximada de 90 metros ( 3 arcos de graus) disponibilizados pela National Aeronautics and Space Administration (NASA), os quais também estão disponíveis com resolução aproximada de 30 metros ( 1 arco de grau) a partir de 2015.

Porém, é necessário lembrar que os modelos digitais de elevação estão sujeitos a distorções. Consequentemente, os atributos topográficos derivados apresentam limitações e nem sempre traduzem as informações fidedignas do terreno, em função de um conjunto de fatores de interferência. As resoluções horizontais e verticais dos dados de elevação utilizados para retratar uma superfície de terreno têm influência significativa no detalhamento e na qualidade das representações, levando vários autores a examinarem os efeitos da resolução espacial sobre o valor e a acurácia dos atributos topográficos, derivados de conjuntos de dados de elevação de resoluções diferentes (ZHANG E MONTGOMERY, 1994; FLORINSKY, 1998; JONES, 1998; WILSON et al., 2000;. Thompson et al., 2001;. TANG et al., 2002;. ZHOU e LIU, 2004; KIENZLE, 2004; WARREN et al., 2004; RAAFLAUB e COLLINS, 2006).

Apesar de amplamente utilizados, muitos modelos são empregados sem considerar a escala como fator que influencia nos resultados dos produtos obtidos a partir destes. Este fator é de elevada relevância já que todas as medidas 


\section{ANÁLISE DA INFLUÊNCIA DA ESCALA NA OBTENÇÃO DOS ATRIBUTOS TOPOGRÁFICOS DERIVADOS DE MDE}

variam em função da escala adotada (EVANS, 1972), isto se deve ao fato da resolução espacial ser diretamente relacionada à resolução do modelo (SHARY et al., 2002) interferindo na aplicação das funções polinomiais tornando sua representação um desafio (LI et al.,2010).

De acordo com Schmidt e Andrew (2005), a superfície da terra é hierárquica e pode ser representada de forma diferente dependendo da escala utilizada (ex: uma vertente convexa pode estar encaixada em uma vertente côncava, que por sua vez pode estar incorporada a um vale).

Este trabalho propende comparar os atributos topográficos derivados das bases cartográficas mais utilizadas no estado do Paraná, contemplando as escalas 1:10000, 1:50000, além dos dados em escala aproximada de 1:100000 derivado dos modelos SRTM. Ressalta-se que os modelos e as bases cartográficas utilizadas são disponibilizados sem custos pelas instituições financiadoras ou executoras. Estes dados serão comparados a uma base de dados inferida com maior resolução espacial, obtida com tecnologia laser scan.

Os atributos analisados foram obtidos a partir de MDEs gerados de formas e dados distintos resultando em modelos com resoluções diferentes. Foram verificados e comparados os atributos topográficos: elevação, declividade, plano e perfil de curvatura. Na comparação para análise dos dados foi utilizada a regressão linear e a matriz de confusão.

Para a realização das comparações, foi escolhido o recorte localizado no município de Almirante Tamandaré, na Região Metropolitana de Curitiba, com área de 72,18ha (Figura 1), inserida no Primeiro Planalto Paranaense (MAACK, 1968).

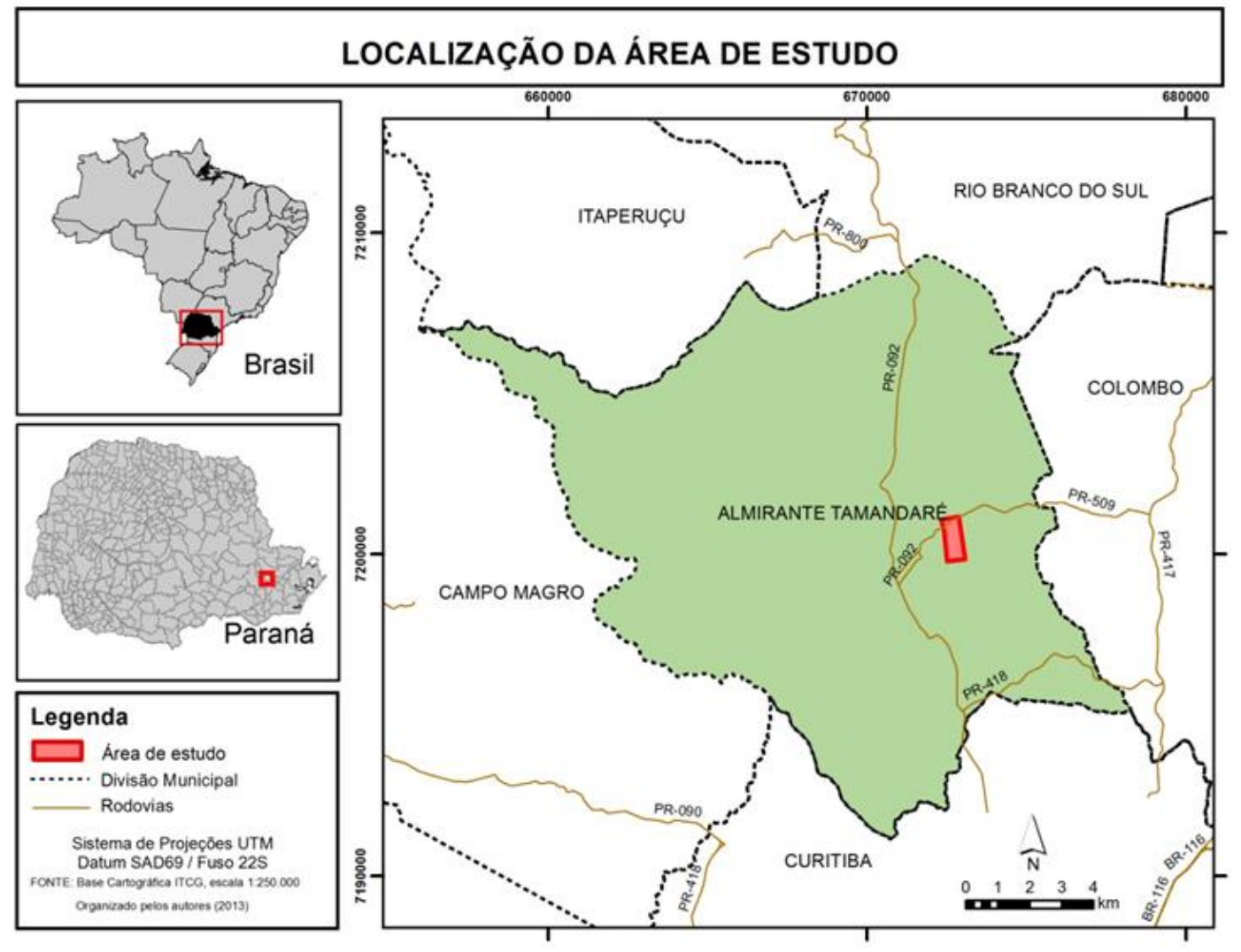

Figura 1 - Localização da área de estudo.

\section{MATERIAL E MÉTODOS}

Foram testados os atributos derivados de cinco modelos digitais de elevação, sendo: a) 02 modelos derivados de interpolação de dados extraídos de bases cartográficas 


\section{ANÁLISE DA INFLUÊNCIA DA ESCALA NA OBTENÇÃO DOS ATRIBUTOS TOPOGRÁFICOS DERIVADOS DE MDE}

planialtimétricas nas escalas 1:10000 e 1:50000.

b) 02 modelos derivados do projeto SRTM (2000), com pixels de $30 \mathrm{~m}$ e $90 \mathrm{~m}$.

c) 01 modelo Laser Scan, o qual foi gerado e avaliado em pesquisa anterior por STABACH (2013).

O primeiro modelo adquirido das bases cartográficas foi obtido das cartas topográficas na escala 1:10000, folha D13NED da bacia do Alto Iguaçu, elaboradas pela Suderhsa (2000) e disponibilizadas pelo Instituto das Águas do Paraná (Águas Paraná). Esta base é composta de temas gerados a partir da restituição digital aerofotogramétrica, complementados nas áreas urbanas com dados de outros projetos existentes no Estado.

O segundo modelo foi gerado a partir da altimetria da Folha MI2842-3 do mapeamento topográfico sistemático na escala 1:50000, disponibilizado pelo Instituto Brasileiro de Geografia e Estatística (IBGE, 1992). Atualmente é uma das escalas que mais tem aplicabilidade considerando seus diferentes usos para mapeamento sistemático, representando a maior quantidade de cartas produzidas nos últimos 20 anos (CAMBOIM et al, 2008).

Para a geração dos MDEs utilizou-se o algoritmo ANUDEM (Topo To Raster), proposto por Hutchinson (1988), o qual combina métodos que utilizam interpolação local e global (ESRI, 1993). Este procedimento permite que arquivos, como curvas de níveis, cursos de rios e pontos cotados, possam ser utilizados durante a interpolação, permitindo a geração de uma rede de drenagem, eliminando as depressões, garantindo uma boa representação do fluxo gravitacional.

Foram utilizados valores de tamanho de pixel diferentes para cada uma das bases cartográficas sendo arbitrado o valor de $1 \mathrm{~m}$ para a geração do MDE derivado do Laser Scan, $5 \mathrm{~m}$ para o MDE derivado da altimetria das cartas $1: 10000,15 \mathrm{~m}$ para o MDE derivado das cartas 1:50000.

Dos modelos SRTM, um emprega os dados originais, com resolução espacial aproximada de 90 metros (disponibilizada pela NASA, 2000) e o segundo faz parte do projeto TOPODATA, disponibilizado com resolução espacial de aproximadamente 30 metros pelo Instituto Espacial de Pesquisas Espaciais -INPE.

Esta última base de dados é oriunda de uma iniciativa do INPE como forma de refinar a qualidade dos dados disponíveis para o país, através do preenchimento das falhas existentes, posteriormente convertidos e estruturados em colunas $x, y, z$, que constituem um primeiro conjunto de dados, ainda com espaçamento (resolução) de 3 arco-segundos. Deste arquivo foram interpoladas as grades de altitude, em um Modelo Digital de Elevação (MDE) refinado então para 1 arco-segundo (VALERIANO, 2011).

Como tamanho de pixel foram utilizados os valores de $90 \mathrm{~m}$ para os dados originais e $30 \mathrm{~m}$ para os dados interpolados.

De posse dos MDE's foram realizados os processamentos para a obtenção dos atributos topográficos. Para a determinação da declividade foi utilizada uma matriz $3 \times 3$ ao redor de cada um dos pixels, sendo estimadas a partir da primeira derivada de $Z$ com relação ao aspecto, centradas em cada ponto da grade de dados (HORN, 1981).

Os gradientes são delimitados nos sentidos oeste para leste $(x)$ e no sentido sul para norte (y), cujo ângulo é dado em graus pela equação 1 (HORN, 1981).

$\beta=\arctan )$

A inclinação de um ponto no terreno é representada por $B$ e os gradientes de diferenças finitas derivadas representadas por $f_{x}$ e $f_{y}$. O gradiente norte-sul $\left(f_{y}\right)$ é calculado a partir de todas as três colunas na matriz de $3 \times 3$, com a coluna central, sendo ponderados 0 dobro elevado como as duas colunas exteriores. O gradiente leste-oeste $\left(f_{x}\right)$ é calculado de uma maneira semelhante (JENESS, 2012), expressas nas equações 2 e 3 . O valor de $L$ é dado pela resolução espacial da grade regular

$$
\begin{aligned}
& N-S=f_{y=\frac{\left[\left(Z_{7}+2 Z_{8}-Z_{9}\right)-\left(Z_{1}+2 Z_{2}-Z_{3}\right)\right]}{8 L y}} \\
& L-O=f_{x=\frac{\left[\left(Z_{3}+2 Z_{6}-Z_{9}\right)-\left(Z_{1}+2 Z_{4}-Z_{7}\right)\right]}{8 L x}}^{\text {(eq. 2) }}
\end{aligned}
$$

As curvaturas são definidas a partir dos valores das diferenças finitas, nas direções de 


\section{ANÁLISE DA INFLUÊNCIA DA ESCALA NA OBTENÇÃO DOS ATRIBUTOS TOPOGRÁFICOS DERIVADOS DE MDE}

norte-sul e leste-oeste. O perfil de curvatura exprime o comportamento de aceleração e desaceleração de fluxos sobre a superfície topográfica onde valores negativos indicam curvaturas convexas e valores positivos indicam curvaturas côncavas. O plano de curvatura é medido na direção perpendicular à declividade da vertente, exprime a relação de convergência e divergência de fluxos sobre a superfície, valores positivos indicam fluxo convergente e valores negativos indicam vertentes convexas (GROHMANN, 2007).

Para a determinação dos planos e perfis de curvatura foi aplicado o algoritmo de Zevenberg e Thorne (1987), onde a análise da área é realizada através de uma matriz $3 \times 3$, cuja célula central relaciona-se com todos os índices topográficos.

Desse modo, o perfil e o plano de curvatura são determinados pelas equações $4 \mathrm{e}$ 5, respectivamente (ZEVENBERGEN e THORNE, 1987).

Perfil $=\frac{-2\left(D G^{2}+E H^{2}+F G H\right)}{G^{2}+H^{2}} \cdot 100$
Plano $=\frac{-2\left(D H^{2}+E G^{2}+F G H\right)}{G^{2}+H^{2}} \cdot 100$

Sendo as variáveis $D, E, F, G$ e $H$ expressas pelas seguintes equações $6,7,8,9$ e 10 , respectivamente:

$D=\frac{\left[\left(Z_{4}+Z_{6}\right) /\left(2-Z_{5}\right)\right]}{L^{2}}$

$E=\frac{\left[\left(Z_{2}+Z_{8}\right) /\left(2-Z_{5}\right)\right]}{L^{2}}$

(eq. 7)
$F=\frac{\left(-Z_{1}+Z_{3}+Z_{7}-Z_{9}\right)}{4 L^{2}}$

$G=\frac{\left(-Z_{4}+Z_{6}\right)}{2 L}$

$H=\frac{\left(Z_{2}-Z_{8}\right)}{2 L}$

Onde $Z n$ é o valor da elevação apresentado naquele ponto e $\mathrm{L}$ o valor de espaçamento da grade. Os atributos extraídos com estes cálculos foram discretizados em classes para comparação da representação das áreas, além de servirem como parâmetros para a realização da comparação a partir da matriz de confusão.

Os intervalos discretizados para a declividade seguiram a proposta da Empresa Brasileira de Pesquisa Agropecuária (EMBRAPA, 1999), visando utilizar um conjunto de classes usualmente empregadas na literatura: a) Inferiores à 3\%; b) de 3 a $8 \%$; c) 8 a 20\%; d), 20 a 45\%; e) 45 a 75\%; (f) acima de 75\%.

Para a discretização do plano e perfil de curvatura foi utilizado o critério de Pennock et al. (1987), onde os valores inferiores a $-0,1$ representam perfis de curvatura côncavo e planos de curvatura convergentes, valores no intervalo de $-0,1$ à 0,1 caracterizam o perfil com feições lineares e plano com feições retas ou planares, e valores superior a 0,1 remetem a perfil com feições convexas e planos com feições divergentes. Esta discretização indica uma possibilita uma caracterização da área a partir da morfologia do relevo, classificando-os conforme demonstrado na figura 2.

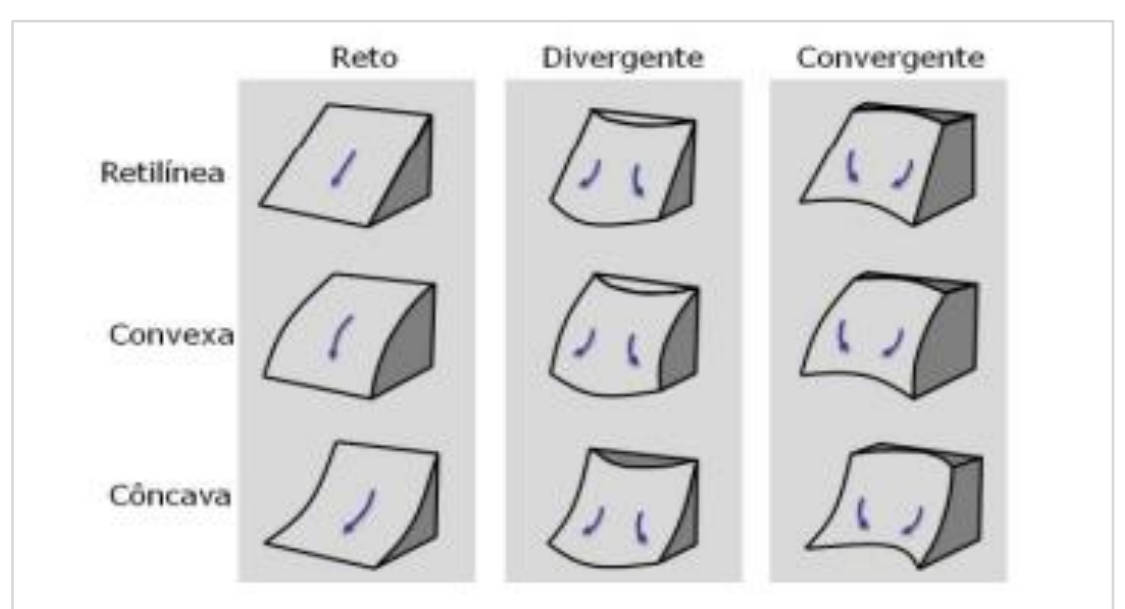

Figura 2 - Curvatura de Plano e Perfil da vertente, indicando o fluxo de água pelas setas.Fonte: Valeriano (2008). 


\section{ANÁLISE DA INFLUÊNCIA DA ESCALA NA OBTENÇÃO DOS ATRIBUTOS TOPOGRÁFICOS DERIVADOS DE MDE}

As bases de dados Laser Scan e SRTM de $90 \mathrm{~m}$ foram escolhidas como parâmetros de correlação, pois representam o menor e o maior tamanho de pixel, além de escalas e formas de obtenção completamente diferentes. A partir disto, houve a aplicação da regressão linear para verificar quais as relações entre os elementos representados.

A Regressão Linear consiste em conhecer o relacionamento entre duas variáveis ( $x$ e y) a partir de uma amostra de observações (JACOBI et al., 2002) ou do universo dos dados.

Como a análise de correlação linear verifica a relação global dos valores dos modelos comparados, fazendo com que, quanto menor a resolução espacial, maior a área do terreno que se deixa de avaliar, foi realizada também a análise a partir da matriz de confusão (CENTENO, 2009).

Centeno (2009) constrói toda uma análise sobre os pressupostos da utilização das matrizes de confusão ou matrizes de erros. Estas matrizes seriam formas de representar as contradições entre a verdade de campo (ou de uma base cartográfica existente) e o resultado do processo de classificação (ou base a ser comparada), ou seja, escolher uma base cartográfica como verdadeira (se considerou como base cartográfica de comparação os dados obtidos com a tecnologia Lidar, os quais possuem maior nível de detalhamento) e confrontá-la com os valores apresentados pela modelagem. Nestas matrizes são analisadas duas situações: as linhas correspondem à classe correta e as colunas as classes estimadas no processo de classificação. Numa situação ideal, onde todos os pixels são classificados corretamente, a matriz vai apresentar valores somente nas diagonais.

A Matriz de Confusão permitiu o cálculo do percentual de concordância entre os elementos representados em cada mapeamento através do valor da exatidão global. Também foi possível avaliar em quanto determinada classe foi subestimada ou superestimada, pois os erros de omissão descrevem a acurácia com que determinada classe de um mapa foi definida, enquanto a taxa de erros de inclusão indica quanto uma classe foi superestimada (SILVEIRA et al., 2012b).

Para a pesquisa assumiu-se que os erros de inclusão representam os pixels que estão na base cartográfica 1:1000 e foram incluídos na classe considerada, sendo identificados pela equação 11 (CENTENO, 2009; SILVEIRA et al., 2012b):

$\operatorname{Ein}(i)=\frac{S c(i)-M(i, i)}{S C(i)}$

Onde Ein(i) significa o erro de inclusão, SC(i) é a soma parcial da coluna i da matriz de erros e $M(i, i)$ são os elementos da diagonal da linha i da matriz de erros.

Os erros de omissão são pixels que pertencem a uma determinada classe que se queira mapear, mas que foram classificados como sendo outra, omitindo-se desta classe, e são representados pela equação 12 (CENTENO, 2009; SILVEIRA et al., 2012b):

$\operatorname{Eom}(i)=\frac{S L(i)-M(i, i)}{S L(i)}$

Onde Eom(i) significa o erro de omissão, SL(i) é a soma parcial da linha i da matriz de erros e $\mathrm{M}(\mathrm{i}, \mathrm{i})$ são os elementos da diagonal da linha i da matriz de erros.

A estimativa global ou exatidão global é o valor que indica a concordância entre os dois mapeamentos usados para a construção da matriz (CENTENO, 2009). Podendo ser calculado como a soma da diagonal principal dividida pelo total de pixels nas amostras, ou seja, a soma de todas as células.

\section{RESULTADOS E DISCUSSÃO}

Os efeitos da alteração no tamanho do pixel adotado são evidenciados, sobretudo nos atributos topográficos derivados, sendo mais difícil sua percepção quando se analisa a variação dos valores hipsométricos.

Se a variação da resolução horizontal do MDE não pressupõe uma interferência significativa nos dados morfométricos, os resultados das correlações lineares (TABELA 1) dos atributos topográficos derivados demonstram o oposto. 
NEUMAN,G., SILVEIRA, C.T., SAMPAIO, T.V.

\section{ANÁLISE DA INFLUÊNCIA DA ESCALA NA OBTENÇÃO DOS ATRIBUTOS TOPOGRÁFICOS DERIVADOS DE MDE}

Tabela 1 - Correlação linear entre os atributos topográficos

\begin{tabular}{c|c|c|c|c|c|c|c|c}
\hline \multicolumn{10}{c|}{ Correção Linear } \\
\hline $\begin{array}{c}\text { Base usada } \\
\text { para }\end{array}$ & \multicolumn{2}{|c|}{ Altimetria } & \multicolumn{2}{c|}{ Declividade } & \multicolumn{2}{l|}{ Plano de Curvatura } & \multicolumn{2}{l|}{ Perfil de Curvatura } \\
\cline { 2 - 10 } comparação & Laser & SRTM & Laser & SRTM & Laser & SRTM & Laser & SRTM \\
& Scan & $90 \mathrm{~m}$ & Scan & $90 \mathrm{~m}$ & Scan & $90 \mathrm{~m}$ & Scan & $90 \mathrm{~m}$ \\
\hline Laser Scan & - & 0,85 & - & 0,3 & - & 0,002 & - & 0,06 \\
\hline Suderhsa & 0,84 & 0,9 & 0,12 & 0,24 & 0,003 & 0,002 & 0,001 & 0,09 \\
\hline IBGE & 0,74 & 0,26 & 0,26 & 0,1 & 0,009 & 0,003 & 0,01 & 0,04 \\
\hline SRTM 30 & 0,87 & 0,99 & 0,11 & 0,96 & 0,0009 & 0,007 & 0,004 & 0,04 \\
\hline SRTM 90 & 0,9 & - & 0,15 & - & 0,0005 & - & 0,007 & - \\
\hline \hline
\end{tabular}

O teste de correlação entre os MDE's resultou em valores, em geral, acima de 0,5, com exceção para a correlação entre os dados da base do IBGE e a SRTM de $90 \mathrm{~m}\left(R^{2}: 0,268\right)$.

O maior valor de correlação encontrado foi entre os modelos SRTM 90m e 30m, o que já era esperado uma vez que uma base é derivada da outra. Chama atenção, contudo, o fato de, mesmo se tratando de produtos homólogos, porém com diferentes resoluções espaciais, há presença de divergências altimétricas significativas, o que reduziu o grau de correlação observado. Isto se dá pelo fato da base SRTM de $30 \mathrm{~m}$ ter sofrido alterações para correção de dados espúrios herdados do modelo original.

Desta forma, mesmo empregando as bases similares para caracterização de uma região, localmente podem ocorrer divergências nos valores altimétricos, alterando também a forma do relevo.

A segunda maior correlação $(0,909)$ foi entre a base SRTM 90m e a Laser Scan (com pixel de $1 \mathrm{~m}$ ). Contudo, a análise do gráfico demonstra que um único valor altimétrico SRTM pode assumir uma grande faixa de valores no modelo Laser Scan, com variações superiores a $20 \mathrm{~m}$. Isto acontece principalmente devido a grande quantidade de pixels, já que na área pertencente a um pixel de $90 \mathrm{~m}$ metros existem diversas altitudes para a base de dados com pixel de $1 \mathrm{~m}$. É possível identificar que as regiões mais planas possuem menores faixas de valores, enquanto que as áreas mais íngremes as faixas são maiores. A correlação não evidencia as discrepâncias altimétricas observadas entre as duas bases, o que é bem observado quando se analisa a correlação do Laser Scan com o SRTM 30m.

Em sentido oposto, a correlação entre o SRTM 90m com a base altimétrica do IBGE apresentou correlação baixa $\left(R^{2}: 0,268\right)$ indicando que os modelos altimétricos descrevem morfologias de relevo completamente distintas para a mesma área de estudo. Cabe ressaltar que esta correlação se refere aos modelos mais usualmente empregados em pesquisas e trabalhos técnicos no Brasil (SOPCHAKI, 2016).

Essa diferença entre os modelos citados pode estar associada à quantidade de informações altimétricas disponíveis na carta do IBGE para subsidiar a aplicação do interpolador. Porém, essa base corresponde ao conjunto de dados topográficos mais empregados no Brasil, uma vez que representa a maior escala disponível para grande parte do território brasileiro. Esta base e a SRTM tem sido amplamente utilizadas, principalmente onde há carência de bases de dados topográficos em escalas maiores, realidade comum de muitas regiões brasileiras.

Para dimensionar espacialmente as divergências de informações entre as bases de dados, foram utilizadas matrizes de confusão para comparação dos valores por classes hipsométricas, conforme quadros 1, 2, 3 e 4, adotando a base Laser Scan como parâmetro de comparação. 
NEUMAN,G., SILVEIRA, C.T., SAMPAIO, T.V.

\section{ANÁLISE DA INFLUÊNCIA DA ESCALA NA OBTENÇÃO DOS ATRIBUTOS TOPOGRÁFICOS DERIVADOS DE MDE}

Quadro 1 - Matriz de confusão - comparação entre os modelos 1:1.000 e 1:10.000

\begin{tabular}{|c|c|c|c|c|c|c|c|c|c|}
\hline \multicolumn{10}{|c|}{ Laser } \\
\hline \multirow{9}{*}{ 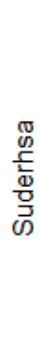 } & Altimetria & 960 & 970 & 980 & 990 & 1000 & 1100 & TOTAL & TOTAL \% \\
\hline & 960 & 2162 & 1694 & 723 & 445 & 128 & 67 & 5219 & 19,3 \\
\hline & 970 & 58 & 1664 & 1243 & 696 & 560 & 336 & 4557 & 16,8 \\
\hline & 980 & 54 & 316 & 976 & 612 & 715 & 880 & 3553 & 13,1 \\
\hline & 990 & 34 & 141 & 611 & 561 & 490 & 1448 & 3285 & 12,1 \\
\hline & 1000 & 31 & 42 & 135 & 436 & 428 & 1970 & 3042 & 11,2 \\
\hline & 1100 & 31 & 48 & 63 & 253 & 573 & 6468 & 7436 & 27,4 \\
\hline & TOTAL & 2370 & 3905 & 3751 & 3003 & 2894 & 11169 & \multirow{2}{*}{27092} & \multirow{2}{*}{100,0} \\
\hline & TOTAL \% & 8,7 & 14,4 & 13,8 & 11,1 & 10,7 & 41,2 & & \\
\hline
\end{tabular}

Quadro 2 - Matriz de confusão - comparação entre os modelos 1:1.000 e 1:50.000

\begin{tabular}{|c|c|c|c|c|c|c|c|c|c|}
\hline \multicolumn{10}{|c|}{ Laser } \\
\hline \multirow{9}{*}{$\begin{array}{l}\text { U్ } \\
\underline{\underline{O}}\end{array}$} & Altimetria & 960 & 970 & 980 & 990 & 1000 & 1100 & TOTAL & TOTAL $\%$ \\
\hline & 960 & 2 & 62 & 28 & 55 & 50 & 19 & 216 & 7,2 \\
\hline & 46 & 41 & 76 & 44 & 21 & 34 & 51 & 267 & 8,9 \\
\hline & 980 & 46 & 99 & 88 & 22 & 27 & 212 & 494 & 16,5 \\
\hline & 990 & 27 & 73 & 60 & 62 & 41 & 139 & 402 & 13,5 \\
\hline & 1000 & 44 & 48 & 62 & 30 & 35 & 137 & 356 & 11,9 \\
\hline & 1100 & 97 & 69 & 132 & 149 & 131 & 674 & 1252 & 41,9 \\
\hline & TOTAL & 257 & 427 & 414 & 339 & 318 & 1232 & \multirow{2}{*}{2987} & \multirow{2}{*}{100,0} \\
\hline & TOTAL $\%$ & 8,6 & 14,3 & 13,9 & 11,3 & 10,6 & 41,2 & & \\
\hline
\end{tabular}

Quadro 3 - Matriz de confusão - comparação entre os modelos 1:1.000 e, pixel de 30m.

\begin{tabular}{|c|c|c|c|c|c|c|c|c|c|}
\hline \multicolumn{10}{|c|}{ Laser } \\
\hline \multirow{9}{*}{ 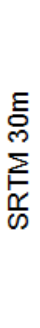 } & Altimetria & 960 & 970 & 980 & 990 & 1000 & 1100 & TOTAL & TOTAL \% \\
\hline & 960 & 1 & 0 & 0 & 0 & 0 & 0 & 1 & 0,1 \\
\hline & 46 & 53 & 35 & 19 & 4 & 0 & 0 & 111 & 14,8 \\
\hline & 980 & 8 & 48 & 31 & 21 & 10 & 0 & 118 & 15,7 \\
\hline & 990 & 2 & 16 & 42 & 27 & 17 & 16 & 120 & 16,0 \\
\hline & 1000 & 1 & 3 & 9 & 26 & 31 & 51 & 121 & 16,1 \\
\hline & 1100 & 0 & 0 & 3 & 10 & 23 & 245 & 281 & 37,4 \\
\hline & TOTAL & 65 & 102 & 104 & 88 & 81 & 312 & \multirow{2}{*}{752} & \multirow{2}{*}{100,0} \\
\hline & TOTAL \% & 8,6 & 13,6 & 13,8 & 11,7 & 10,8 & 41,5 & & \\
\hline
\end{tabular}

Quadro 4 - Matriz de confusão - comparação entre os modelos 1:1.000 e pixel de 90m.

\begin{tabular}{|c|c|c|c|c|c|c|c|c|c|}
\hline \multicolumn{10}{|c|}{ Laser } \\
\hline \multirow{9}{*}{ 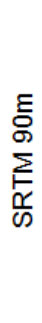 } & Altimetria & 960 & 970 & 980 & 990 & 1000 & 1100 & TOTAL & TOTAL $\%$ \\
\hline & 960 & 1 & 0 & 0 & 0 & 0 & 0 & 1 & 1,1 \\
\hline & 46 & 6 & 6 & 3 & 2 & 0 & 0 & 17 & 18,9 \\
\hline & 980 & 0 & 3 & 5 & 2 & 0 & 0 & 10 & 11,1 \\
\hline & 990 & 0 & 3 & 6 & 3 & 2 & 1 & 15 & 16,7 \\
\hline & 1000 & 0 & 0 & 2 & 3 & 5 & 2 & 12 & 13,3 \\
\hline & 1100 & 0 & 0 & 0 & 1 & 2 & 32 & 35 & 38,9 \\
\hline & TOTAL & 7 & 12 & 16 & 11 & 9 & 35 & \multirow{2}{*}{90} & \multirow{2}{*}{100,0} \\
\hline & TOTAL \% & 7,8 & 13,3 & 17,8 & 12,2 & 10,0 & 38,9 & & \\
\hline
\end{tabular}

Os índices de exatidão global observados foram de 0,45 para o modelo da Suderhsa, de 0,31 para o modelo derivado das cartas do IBGE, 0,49 para o modelo SRTM 30m e 0,58 para a comparação com o modelo SRTM $90 \mathrm{~m}$.

Da mesma forma que as correlações lineares o índice de exatidão não reflete as discrepâncias encontradas entre as bases de dados, isto porque devido ao tamanho de pixel a base de dados SRTM $90 \mathrm{~m}$ possui melhores índices, pois apresenta menor quantidade de pixels para comparação. Observe que quanto maior o pixel melhor a exatidão, com exceção do IBGE, que apresenta problemas derivados da baixa quantidade de informações para aplicação 


\section{ANÁLISE DA INFLUÊNCIA DA ESCALA NA OBTENÇÃO DOS ATRIBUTOS TOPOGRÁFICOS DERIVADOS DE MDE}

do algoritmo de interpolação neste recorte específico.

Estas afirmações são reforçadas pelos erros de omissão e inclusão. Enquanto nas bases de dados com maiores detalhamentos os erros de omissão predominaram, os erros de inclusão predominaram nos modelos com pixels maiores, como o SRTM 30m e SRTM 90m, chegando a valores acima de $73 \%$ de erros de inclusão na segunda base mencionada.
A figura 3 evidencia as áreas nas quais os valores dos pixels foram concordantes. Em geral estas áreas representam as regiões mais elevadas da área de estudo, enquanto que os maiores índices de erros corresponderam às regiões mais baixas do relevo. As células verdes representam o número de células concordantes da mesma classe advinda de MDEs distintos em comparação ao MDE com resolução mais refinada (MDE base Laser Scan).

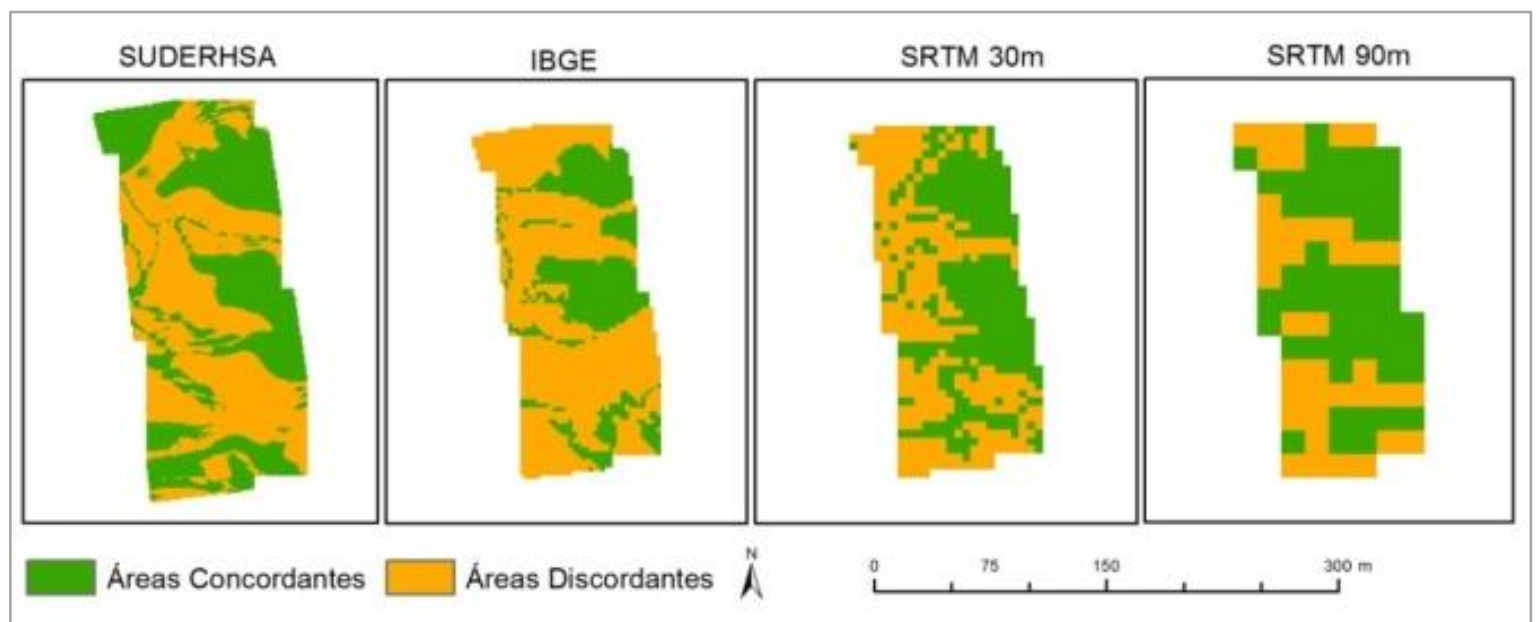

Figura 3 - Áreas concordantes e discordantes dos modelos de elevação

Se por um lado a comparação entre os modelos de elevação resultou em valores de correlação em geral acima de 0,5, a correlação entre os atributos derivados (declividade, plano e perfil de curvatura) resultou em baixos valores de correlação, exceto para as duas bases SRTM que apresentaram correlação de 0,96 no atributo declividade.

A figura 4 representa os perfis do atributo declividade das bases cartográficas em segmentos distintos. O segmento A contempla a região mais íngreme e o segmento $B$ uma faixa de transição para a região mais plana. As variações apresentadas nas representações correspondem a alteração na dimensão do pixel aplicado a superfície pelo modelo decompondo os dados mais refinados em todas as escalas, sendo maior este efeito nos modelos com menor detalhamento, seja advindo da escala ou do tamanho de pixel utilizado. 


\section{ANÁLISE DA INFLUÊNCIA DA ESCALA NA OBTENÇÃO DOS ATRIBUTOS TOPOGRÁFICOS} DERIVADOS DE MDE

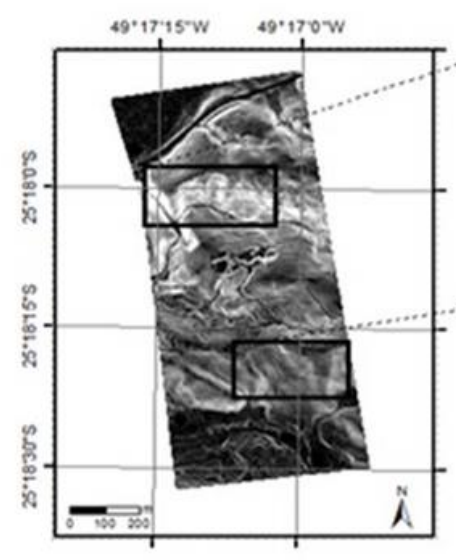

Dados referentes ao segmento $A$
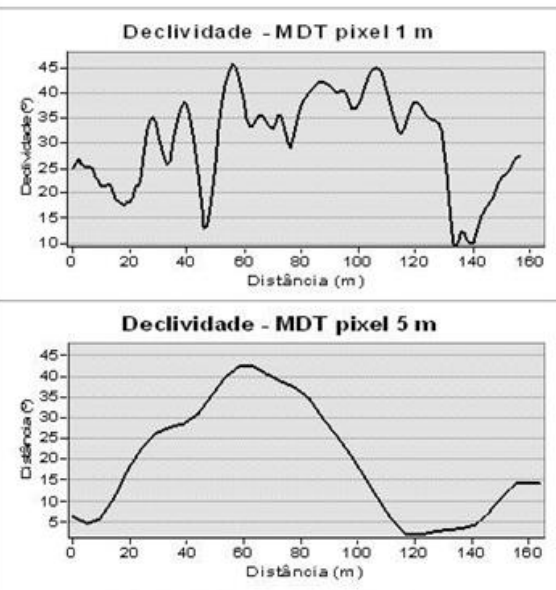

Declividade - MDT pixel $15 \mathrm{~m}$
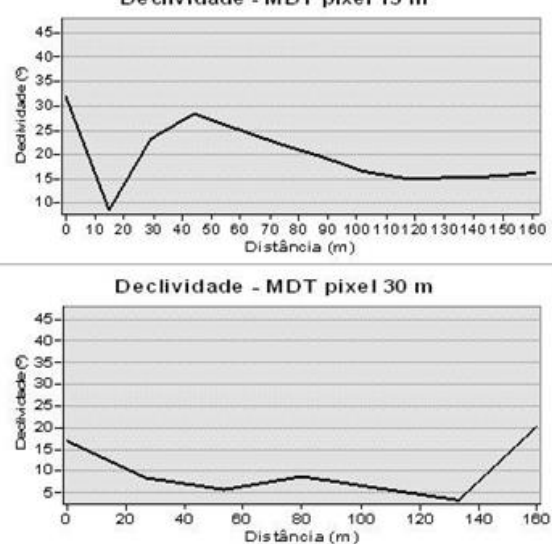

Declividade - MDT pixel $90 \mathrm{~m}$

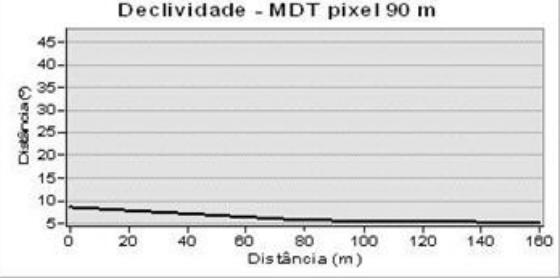

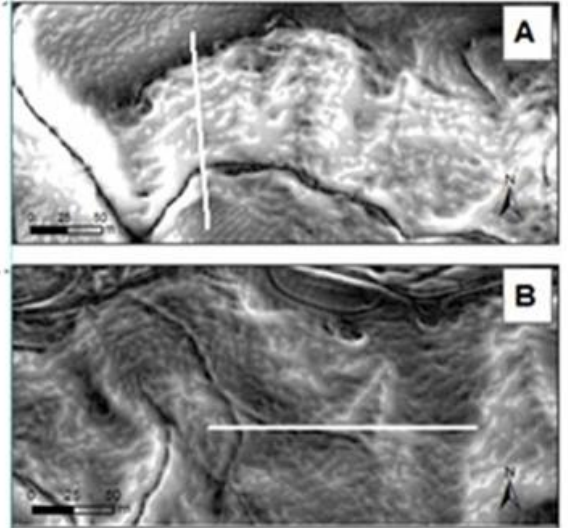

Dados referentes ao segmento B
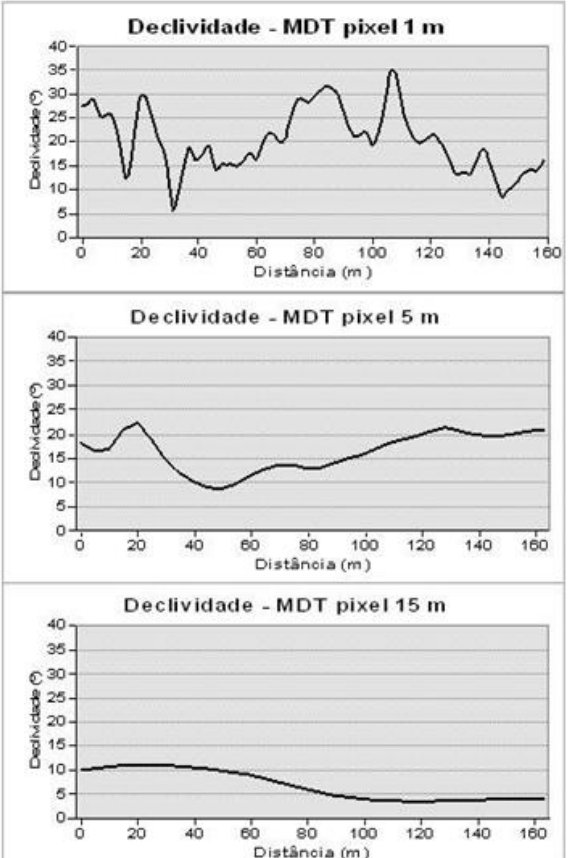

Declividade - MDT pixel $30 \mathrm{~m}$
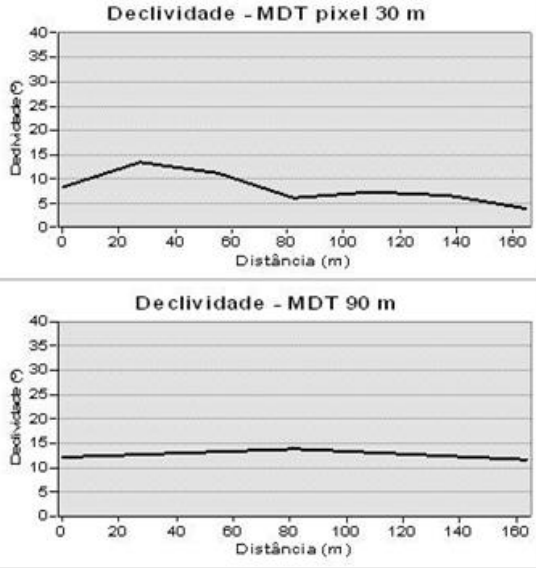

Figura 4 - Perfis de declividade dos segmentos A (à esquerda) e B (à direita). 


\section{ANÁLISE DA INFLUÊNCIA DA ESCALA NA OBTENÇÃO DOS ATRIBUTOS TOPOGRÁFICOS DERIVADOS DE MDE}

Os modelos obtidos a partir da base Laser Scan apresentam pixels em todas as classes de declividade, inclusive aquelas acima de $75 \%$, enfatizando sua capacidade de representar pequenas alterações nas feições do relevo.
Este detalhamento pode ser observado tanto em perfil (Figura 4), quanto na forma de área (Figura 5), que destaca a declividade da porção norte da área de estudo

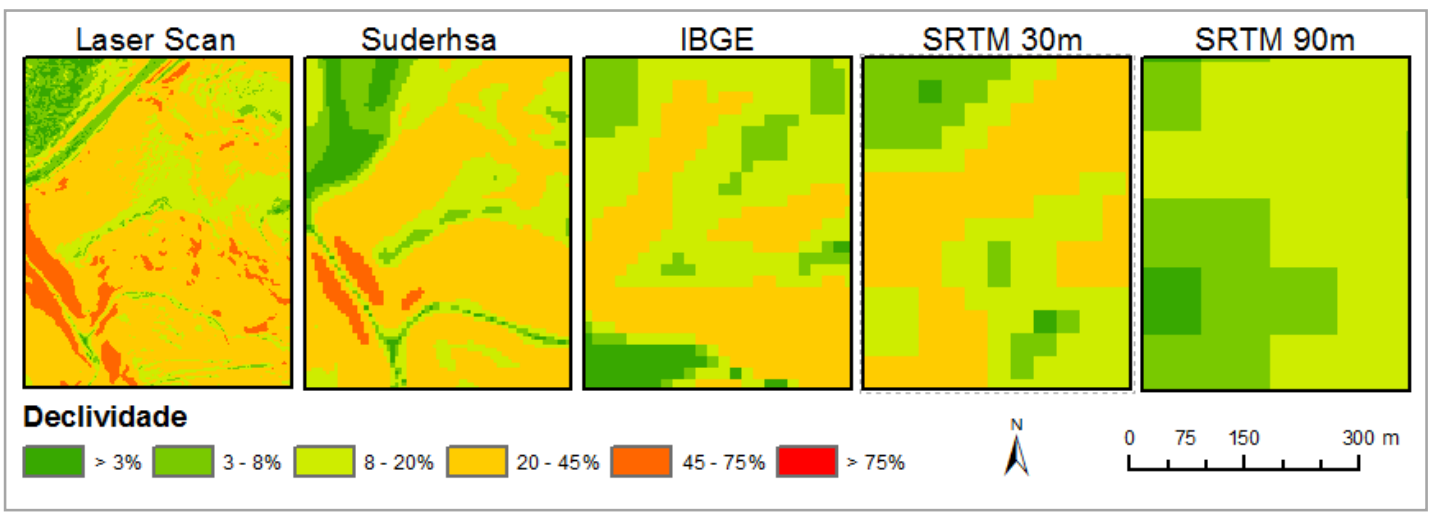

Figura 5 - Declividade da porção norte da área de estudo

O detalhamento promovido pelo aumento na resolução resultou em um maior número de pixels nas classes de declividade de 45\%a $75 \%$ e acima de $75 \%$, enquanto modelos com menores resoluções espaciais obtiveram menor representatividade (Figura 6).

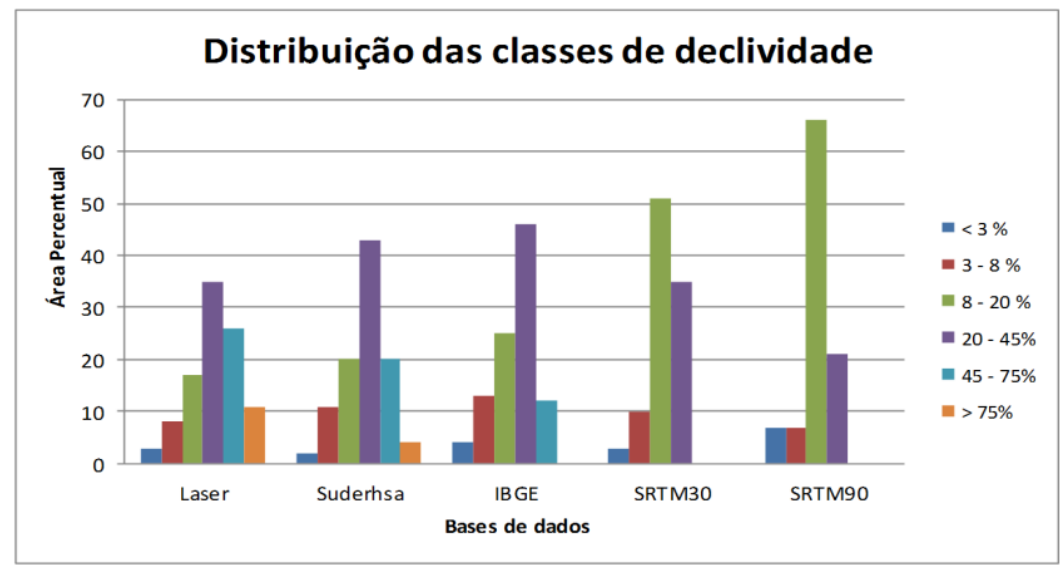

Figura 6 - Gráfico com a distribuição das classes de declividade obtidas de diferentes MDE's.

A quantidade de pixels na classe de declividade de $8 \%$ a $20 \%$ aumenta na medida em que se reduz a resolução dos MDEs. O mesmo não ocorre para a classe de $3 \%$ a $8 \%$. Classes com maiores valores de declividade (45\% a $>75 \%)$ apresentam redução no número de pixels, e sequer aparecem nos modelos de menor resolução.
Do mesmo modo que para a correlação da hipsometria, os atributos declividade, plano e perfil de curvatura também foram correlacionados com os dados da base Laser Scan com a aplicação das matrizes de confusão. Os quadros 5, 6, 7 e 8 apresentam as matrizes do atributo declividade. 
NEUMAN,G., SILVEIRA, C.T., SAMPAIO, T.V.

\section{ANÁLISE DA INFLUÊNCIA DA ESCALA NA OBTENÇÃO DOS ATRIBUTOS TOPOGRÁFICOS DERIVADOS DE MDE}

Quadro 5 - Matriz de confusão - comparação entre as resoluções 1.000 e 1:10.000

\begin{tabular}{|c|c|c|c|c|c|c|c|c|c|}
\hline \multicolumn{10}{|c|}{ Laser } \\
\hline \multirow{9}{*}{ 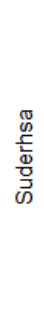 } & $\begin{array}{c}\text { Classes } \\
\text { Declividade }\end{array}$ & $0-3$ & $3-8$ & $8-20$ & $20-45$ & $45-75$ & $>75$ & TOTAL & TOTAL \% \\
\hline & $0-3$ & 14 & 48 & 158 & 135 & 106 & 24 & 485 & 1,8 \\
\hline & $3-8$ & 295 & 833 & 859 & 557 & 337 & 76 & 2957 & 10,9 \\
\hline & $8-20$ & 316 & 704 & 1206 & 1751 & 976 & 261 & 5214 & 19,3 \\
\hline & $20-45$ & 99 & 494 & 1702 & 5148 & 3177 & 1100 & 11720 & 43,3 \\
\hline & $45-75$ & 20 & 103 & 509 & 1805 & 1956 & 1238 & 5631 & 20,8 \\
\hline & $>75$ & 2 & 6 & 29 & 266 & 419 & 354 & 1076 & 4,0 \\
\hline & TOTAL & 746 & 2188 & 4463 & 9662 & 6971 & 3053 & \multirow{2}{*}{27083} & \multirow{2}{*}{100,0} \\
\hline & TOTAL \% & 2,8 & 8,1 & 16,5 & 35,7 & 25,7 & 11,3 & & \\
\hline
\end{tabular}

Quadro 6 - Matriz de confusão - comparação entre os modelos 1:1.000 e 1:50.000

\begin{tabular}{|c|c|c|c|c|c|c|c|c|c|}
\hline \multicolumn{10}{|c|}{ Laser } \\
\hline \multirow{9}{*}{$\begin{array}{l}\text { U్ } \\
\underline{\underline{\underline{M}}}\end{array}$} & $\begin{array}{c}\text { Classes } \\
\text { Declividade }\end{array}$ & $0-3$ & $3-8$ & $8-20$ & $20-45$ & $45-75$ & $>75$ & TOTAL & TOTAL \% \\
\hline & $0-3$ & 1 & 4 & 22 & 31 & 34 & 10 & 102 & 3,4 \\
\hline & $3-8$ & 9 & 30 & 57 & 162 & 89 & 25 & 372 & 12,5 \\
\hline & $8-20$ & 33 & 58 & 135 & 292 & 178 & 66 & 762 & 25,6 \\
\hline & $20-45$ & 41 & 121 & 244 & 405 & 373 & 180 & 1364 & 45,9 \\
\hline & $45-75$ & 5 & 14 & 43 & 162 & 96 & 51 & 371 & 12,5 \\
\hline & $>75$ & 0 & 1 & 1 & 0 & 0 & 0 & 2 & 0,1 \\
\hline & TOTAL & 89 & 228 & 502 & 1052 & 770 & 332 & \multirow{2}{*}{2973} & \multirow{2}{*}{100,0} \\
\hline & TOTAL \% & 3,0 & 7,7 & 16,9 & 35,4 & 25,9 & 11,2 & & \\
\hline
\end{tabular}

Quadro 7 - Matriz de confusão - comparação entre os modelos 1:1.000 e com pixel de 30m.

\begin{tabular}{|c|c|c|c|c|c|c|c|c|c|}
\hline \multicolumn{10}{|c|}{ Laser } \\
\hline \multirow{9}{*}{ 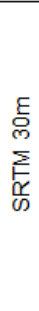 } & $\begin{array}{c}\text { Classes } \\
\text { Declividade }\end{array}$ & $0-3$ & $3-8$ & $8-20$ & $20-45$ & $45-75$ & $>75$ & TOTAL & TOTAL \% \\
\hline & $0-3$ & 1 & 8 & 8 & 8 & 1 & 1 & 27 & 3,6 \\
\hline & $3-8$ & 9 & 15 & 26 & 20 & 7 & 2 & 79 & 10,5 \\
\hline & $8-20$ & 9 & 21 & 50 & 140 & 110 & 44 & 374 & 49,9 \\
\hline & $20-45$ & 0 & 12 & 32 & 106 & 84 & 36 & 270 & 36,0 \\
\hline & $45-75$ & 0 & 0 & 0 & 0 & 0 & 0 & 0 & 0,0 \\
\hline & $>75$ & 0 & 0 & 0 & 0 & 0 & 0 & 0 & 0,0 \\
\hline & TOTAL & 19 & 56 & 116 & 274 & 202 & 83 & \multirow{2}{*}{750} & \multirow{2}{*}{100,0} \\
\hline & TOTAL $\%$ & 2,5 & 7,5 & 15,5 & 36,5 & 26,9 & 11,1 & & \\
\hline
\end{tabular}

Quadro 8 - Matriz de confusão - comparação entre os modelos 1:1.000 e pixel de 90m.

\begin{tabular}{|c|c|c|c|c|c|c|c|c|c|}
\hline \multicolumn{10}{|c|}{ Laser } \\
\hline \multirow{9}{*}{ 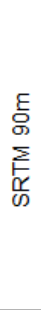 } & $\begin{array}{c}\text { Classes } \\
\text { Declividade }\end{array}$ & $0-3$ & $3-8$ & $8-20$ & $20-45$ & $45-75$ & $>75$ & TOTAL & TOTAL \% \\
\hline & $0-3$ & 0 & 1 & 4 & 1 & 0 & 0 & 6 & 6,7 \\
\hline & $3-8$ & 2 & 0 & 2 & 1 & 1 & 0 & 6 & 6,7 \\
\hline & $8-20$ & 2 & 1 & 6 & 24 & 19 & 7 & 59 & 65,6 \\
\hline & $20-45$ & 0 & 0 & 1 & 8 & 8 & 2 & 19 & 21,1 \\
\hline & $45-75$ & 0 & 0 & 0 & 0 & 0 & 0 & 0 & 0,0 \\
\hline & $>75$ & 0 & 0 & 0 & 0 & 0 & 0 & 0 & 0,0 \\
\hline & TOTAL & 4 & 2 & 13 & 34 & 28 & 9 & \multirow{2}{*}{90} & \multirow{2}{*}{100,0} \\
\hline & TOTAL \% & 4,4 & 2,2 & 14,4 & 37,8 & 31,1 & 10,0 & & \\
\hline
\end{tabular}

Os erros de omissão foram maiores para os modelos com maior resolução (pixel de 1, 5 e $10 \mathrm{~m})$, tendo o modelo advindo da base do IBGE a quantidade de $65 \%$ de seus erros em forma de erros de omissão, devido às falhas para classificação da primeira classe utilizada (0 a 3\%).
Os erros de inclusão concentraram-se nas bases SRTM chegando a $100 \%$ de erros de inclusão nas classes de 45 a $>75 \%$ nos dois modelos.

A figura 7 representa espacialmente as áreas concordantes e discordantes entre $\mathrm{o}$ modelo laser e os demais modelos. 


\section{ANÁLISE DA INFLUÊNCIA DA ESCALA NA OBTENÇÃO DOS ATRIBUTOS TOPOGRÁFICOS DERIVADOS DE MDE}

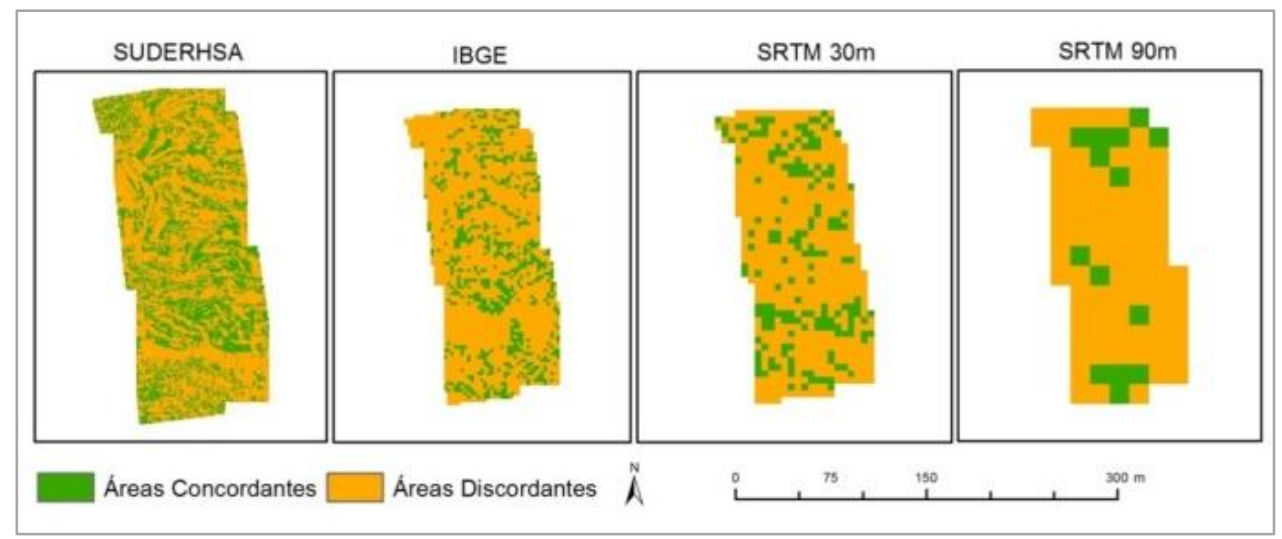

Figura 7 - Áreas concordantes e discordantes do atributo declividade

No caso das curvaturas o efeito do aumento no tamanho do pixel foi intensificado e praticamente impossibilitou as análises a partir das correlações, pois o plano e o perfil de curvatura resultaram em valores sempre abaixo de 0,09 , independente da base considerada, indicando inexistência de relação entre os dados.
As figuras 8 e 9 possibilitam a visualização da distribuição espacial das classes do plano e perfil de curvatura, na porção norte da área de estudo e demonstram a grande diferença entre os modelos.

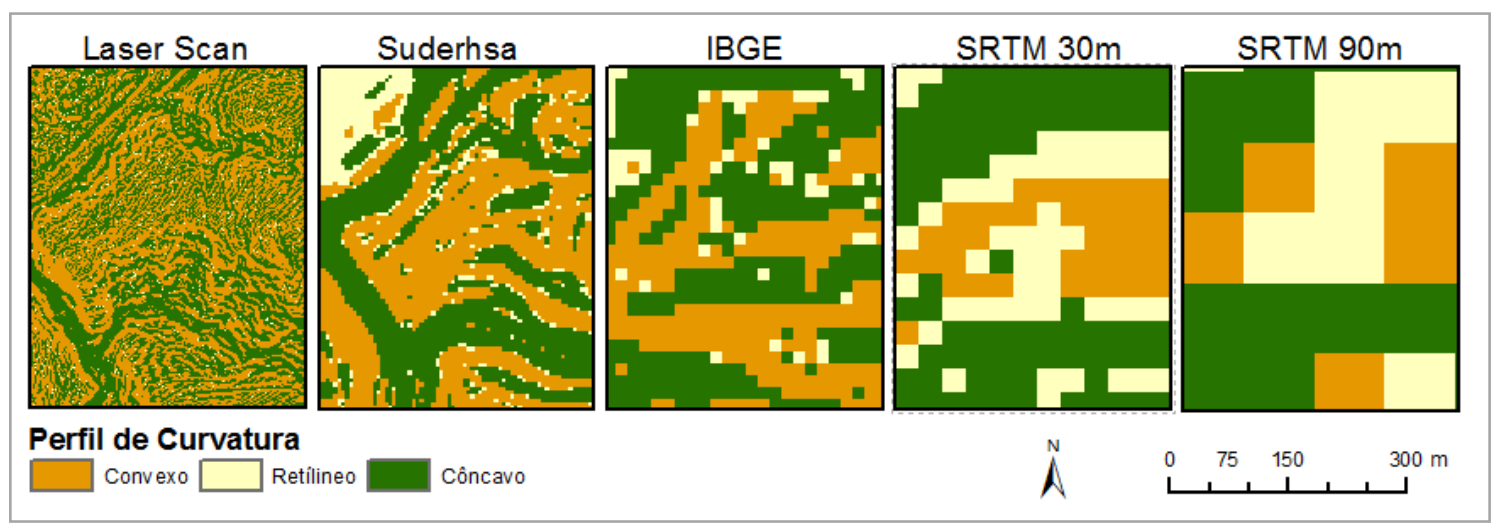

Figura 8 - Perfil de curvatura da porção norte da área de estudo.

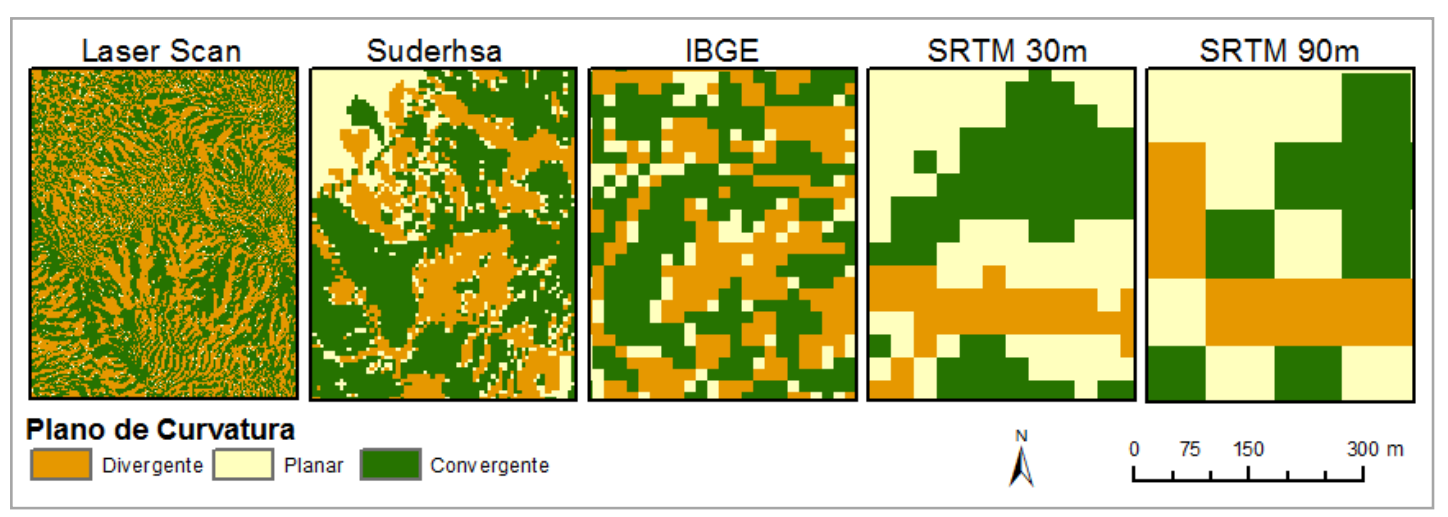

Figura 9 - Plano de curvatura da porção norte da área de estudo.

Neste caso a redução da resolução dos modelos resultou em um aumento das classes retilíneas e planares, enquanto o aumento de resolução indicou tendência de desaparecimento dessa classe, como pode ser observado nos dados da base Laser Scan na figura 10 e 11. 


\section{ANÁLISE DA INFLUÊNCIA DA ESCALA NA OBTENÇÃO DOS ATRIBUTOS TOPOGRÁFICOS DERIVADOS DE MDE}

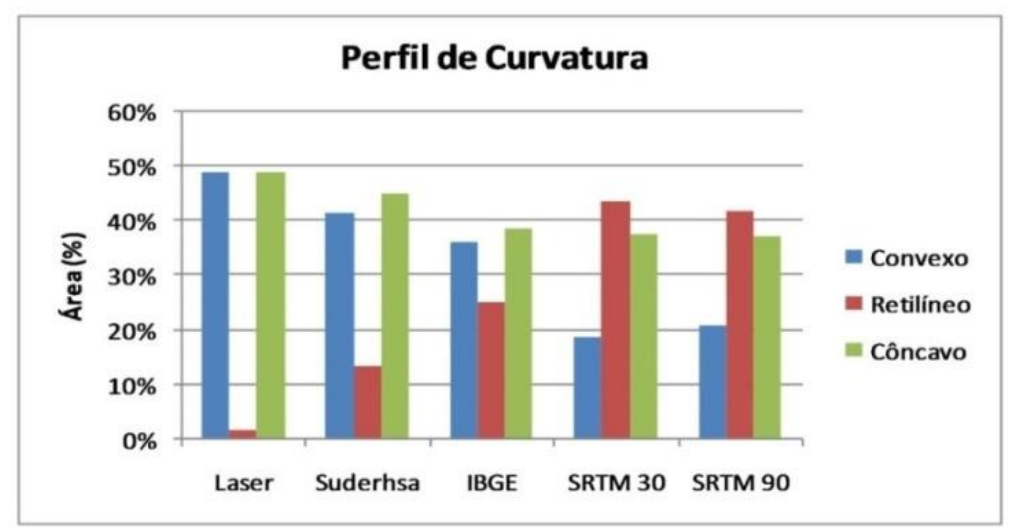

Figura 10 - Gráfico com a distribuição percentual dos dados de plano de curvatura.

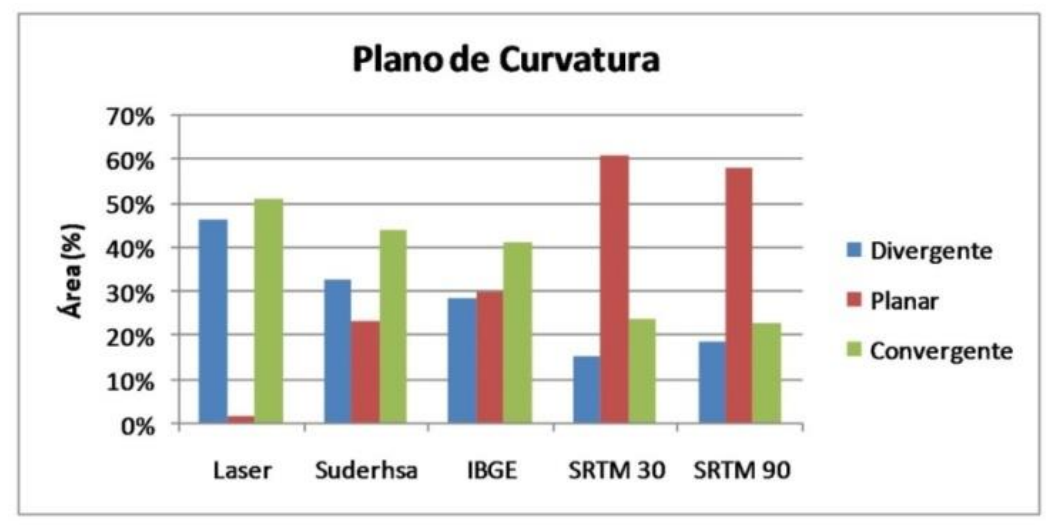

Figura 11 - Gráfico com a distribuição percentual dos dados de perfil de curvatura.

Os quadros 9, 10, 11 e 12 apresentam a quantidade de pixels concordantes entre os dados do plano de curvatura.

Os índices da exatidão global, para este atributo seguiram a mesma lógica que a apresentada pelo atributo declividade, apresentando os índices de 0,39 para a Suderhsa, de 0,35 para o IBGE, de 0,22 para SRTM com pixel $30 \mathrm{~m}$ e de 0,12 para a base SRTM com pixel de $90 \mathrm{~m}$, com a diferença que para este atributo não houve a aglomeração de pixels em determinadas porções específicas da área de estudo.

Quadro 9 - Matriz de confusão - comparação entre os modelos 1:1000 e 1:10.000

\begin{tabular}{|c|c|c|c|c|c|c|}
\hline \multicolumn{7}{|c|}{ Laser } \\
\hline \multirow{6}{*}{ 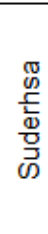 } & Classes & Divergente & Planar & Convergente & TOTAL & TOTAL \% \\
\hline & Divergente & 4222 & 182 & 4656 & 9060 & 33,4 \\
\hline & Planar & 2787 & 147 & 3146 & 6080 & 22,4 \\
\hline & Convergente & 5497 & 241 & 6214 & 11952 & 44,1 \\
\hline & TOTAL & 12506 & 570 & 14016 & ?709? & 1000 \\
\hline & TOTAL \% & 46,2 & 2,1 & 51,7 & & \\
\hline
\end{tabular}




\section{ANÁLISE DA INFLUÊNCIA DA ESCALA NA OBTENÇÃO DOS ATRIBUTOS TOPOGRÁFICOS DERIVADOS DE MDE}

Quadro 10 - Matriz de confusão - comparação entre os modelos 1:1000 e 1:50.000

\begin{tabular}{|c|c|c|c|c|c|c|}
\hline \multicolumn{7}{|c|}{ Laser } \\
\hline \multirow{6}{*}{ 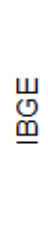 } & Classes & Divergente & Planar & Convergente & TOTAL & TOTAL \% \\
\hline & Divergente & 425 & 11 & 478 & 914 & 30,6 \\
\hline & Planar & 376 & 17 & 456 & 849 & 28,4 \\
\hline & Convergente & 566 & 35 & 623 & 1224 & 41,0 \\
\hline & TOTAL & 1367 & 63 & 1557 & \multirow{2}{*}{2987} & \multirow{2}{*}{100,0} \\
\hline & TOTAL \% & 45,8 & 2,1 & 52,1 & & \\
\hline
\end{tabular}

Quadro 11 - Matriz de confusão - comparação entre os dados 1:1000 e pixel de 30m.

\begin{tabular}{|c|c|c|c|c|c|c|}
\hline \multicolumn{7}{|c|}{ Laser } \\
\hline \multirow{6}{*}{ 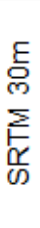 } & Classes & Divergente & Planar & Convergente & TOTAL & TOTAL \% \\
\hline & Divergente & 60 & 2 & 66 & 128 & 17,0 \\
\hline & Planar & 181 & 12 & 243 & 436 & 58,0 \\
\hline & Convergente & 89 & 4 & 95 & 188 & 25,0 \\
\hline & TOTAL & 330 & 18 & 404 & \multirow{2}{*}{752} & \multirow{2}{*}{100,0} \\
\hline & TOTAL \% & 43,9 & 2,4 & 537 & & \\
\hline
\end{tabular}

Quadro 10 - Matriz de confusão - comparação entre os dados 1:1000 e pixel de 90m.

\begin{tabular}{|c|c|c|c|c|c|c|}
\hline \multicolumn{7}{|c|}{ Laser } \\
\hline \multirow{6}{*}{ 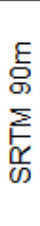 } & Classes & Divergente & Planar & Convergente & TOTAL & TOTAL $\%$ \\
\hline & Divergente & 4 & 0 & 9 & 13 & 14,4 \\
\hline & Planar & 25 & 1 & 36 & 62 & 68,9 \\
\hline & Convergente & 9 & 0 & 6 & 15 & 16,7 \\
\hline & TOTAL & 38 & 1 & 51 & \multirow{2}{*}{90} & \multirow{2}{*}{100,0} \\
\hline & TOTAL \% & 42,2 & 1.1 & 56,7 & & \\
\hline
\end{tabular}

Os erros de omissão foram altos para a classe planar, chegando em $98,6 \%$ na comparação com os dados IBGE. Os erros de inclusão foram maiores nas bases de dados SRTM, chegando a $76 \%$ na classe divergente da base SRTM $90 \mathrm{~m}$ e resultando na maior incompatibilidade com todos os outros modelos.

\section{A figura 12 representa espacialmente} onde se encontram as áreas concordantes e as áreas discordantes entre o modelo laser e os demais modelos.

Os quadros 13, 14, 15 e 16 destacam em azul a quantidade de pixels das áreas concordantes para o atributo perfil de curvatura.

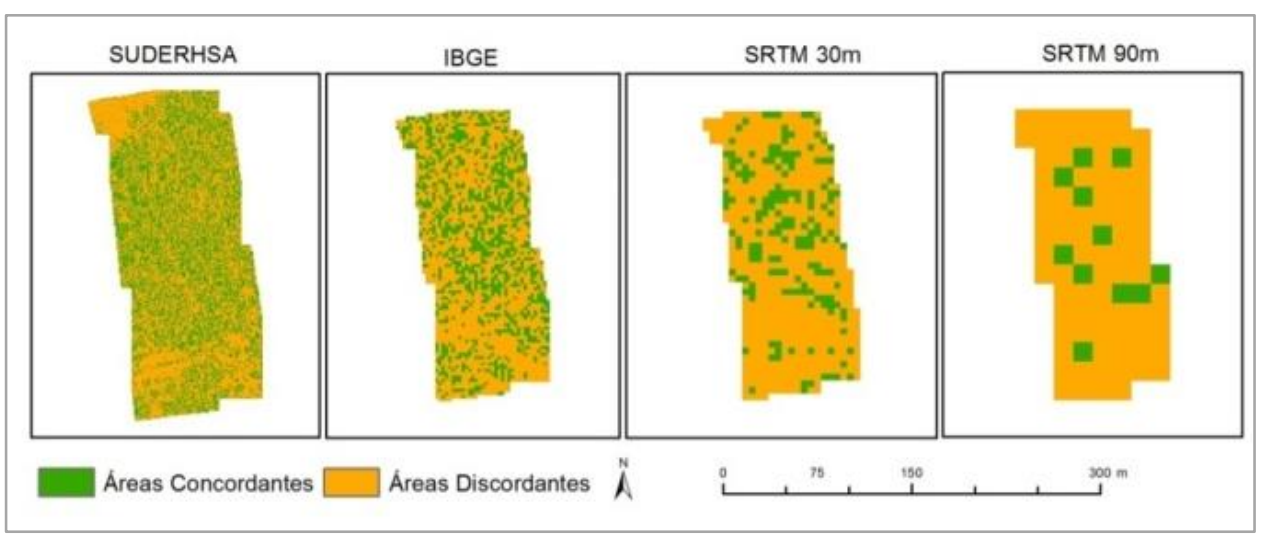

Figura 12 - Áreas concordantes e discordantes do atributo plano de curvatura 


\section{ANÁLISE DA INFLUÊNCIA DA ESCALA NA OBTENÇÃO DOS ATRIBUTOS TOPOGRÁFICOS DERIVADOS DE MDE}

Quadro 13 - Matriz de confusão - comparação entre os modelos 1:1.000 e 1:10.000

\begin{tabular}{|c|c|c|c|c|c|c|}
\hline \multicolumn{7}{|c|}{ Laser } \\
\hline \multirow{6}{*}{ 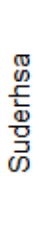 } & Classes & Convexo & Retilineo & Côncavo & TOTAL & TOTAL \% \\
\hline & Convexo & 5688 & 183 & 5500 & 11371 & 42,0 \\
\hline & Retílineo & 1672 & 84 & 1783 & 3539 & 13,1 \\
\hline & Côncavo & 5981 & 222 & 5979 & 12182 & 45,0 \\
\hline & TOTAL & 13341 & 489 & 13262 & \multirow{2}{*}{27092} & \multirow{2}{*}{100,0} \\
\hline & TOTAL \% & 49,2 & 1,8 & 49,0 & & \\
\hline
\end{tabular}

Quadro 14 - Matriz de confusão - comparação entre os modelos 1:1.000 e 1:50.000

\begin{tabular}{|c|c|c|c|c|c|c|}
\hline \multicolumn{7}{|c|}{ Laser } \\
\hline \multirow{6}{*}{$\begin{array}{l}\underset{\bigcup}{0} \\
\underline{\underline{M}}\end{array}$} & Classes & Convexo & Retilíneo & Côncavo & TOTAL & TOTAL \% \\
\hline & Convexo & 552 & 18 & 510 & 1080 & 36,2 \\
\hline & Retilíneo & 337 & 10 & 345 & 692 & 23,2 \\
\hline & Côncavo & 611 & 18 & 586 & 1215 & 40,7 \\
\hline & TOTAL & 1500 & 46 & 1441 & \multirow{2}{*}{2987} & \multirow{2}{*}{100,0} \\
\hline & TOTAL \% & 50,2 & 1,5 & 48,2 & & \\
\hline
\end{tabular}

Quadro 15 - Matriz de confusão - comparação entre os modelos 1:1.000 e pixel de 30m.

\begin{tabular}{|c|c|c|c|c|c|c|}
\hline \multicolumn{7}{|c|}{ Laser } \\
\hline \multirow{6}{*}{ 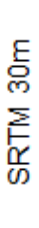 } & Classes & Convexo & Retilíneo & Côncavo & TOTAL & TOTAL \% \\
\hline & Convexo & 89 & 0 & 88 & 177 & 23,5 \\
\hline & Retilíneo & 154 & 7 & 147 & 308 & 41,0 \\
\hline & Côncavo & 119 & 5 & 143 & 267 & 35,5 \\
\hline & TOTAL & 362 & 12 & 378 & \multirow{2}{*}{752} & \multirow{2}{*}{100,0} \\
\hline & TOTAL \% & 48,1 & 1,6 & 50,3 & & \\
\hline
\end{tabular}

Quadro 16 - Matriz de confusão - comparação entre os modelos 1:1.000 e pixel de 90m.

\begin{tabular}{|c|c|c|c|c|c|c|}
\hline \multicolumn{7}{|c|}{ Laser } \\
\hline \multirow{6}{*}{$\begin{array}{l}E \\
\bar{\sigma} \\
\bar{\sigma} \\
\frac{\alpha}{\sigma} \\
0\end{array}$} & Classes & Convexo & Retilíneo & Côncavo & TOTAL & TOTAL \% \\
\hline & Convexo & 13 & 0 & 12 & 25 & 27,8 \\
\hline & Retilíneo & 21 & 0 & 13 & 34 & 37,8 \\
\hline & Côncavo & 20 & 0 & 11 & 31 & 34,4 \\
\hline & TOTAL & 54 & 0 & 36 & \multirow{2}{*}{90} & \multirow{2}{*}{100,0} \\
\hline & TOTAL $\%$ & 60,0 & 0,0 & 40,0 & & \\
\hline
\end{tabular}

O atributo perfil de curvatura também apresentou maior correspondência nos modelos de melhor resolução espacial, possuindo índices de acerto de 0,43 para o modelo derivado da base da Suderhsa, 0,38 para o modelo derivado da base do IBGE, 0,31 para o modelo SRTM de $30 \mathrm{~m}$ e 0,26 para o modelo SRTM com pixel de $90 \mathrm{~m}$.

O cálculo dos atributos de segunda derivada com diferentes tamanhos de pixel demonstrou baixos valores de correlação lineares e altos valores de erros de omissão que chegam a $100 \%$ na categoria planar da base de dados SRTM $90 \mathrm{~m}$ e $79 \%$ de erros de inclusão na categoria planar da base de dados Laser Scan.

A figura 15 apresenta em laranja a área correspondente aos pixels concordantes entre o modelo laser e os demais modelos. 


\section{ANÁLISE DA INFLUÊNCIA DA ESCALA NA OBTENÇÃO DOS ATRIBUTOS TOPOGRÁFICOS DERIVADOS DE MDE}

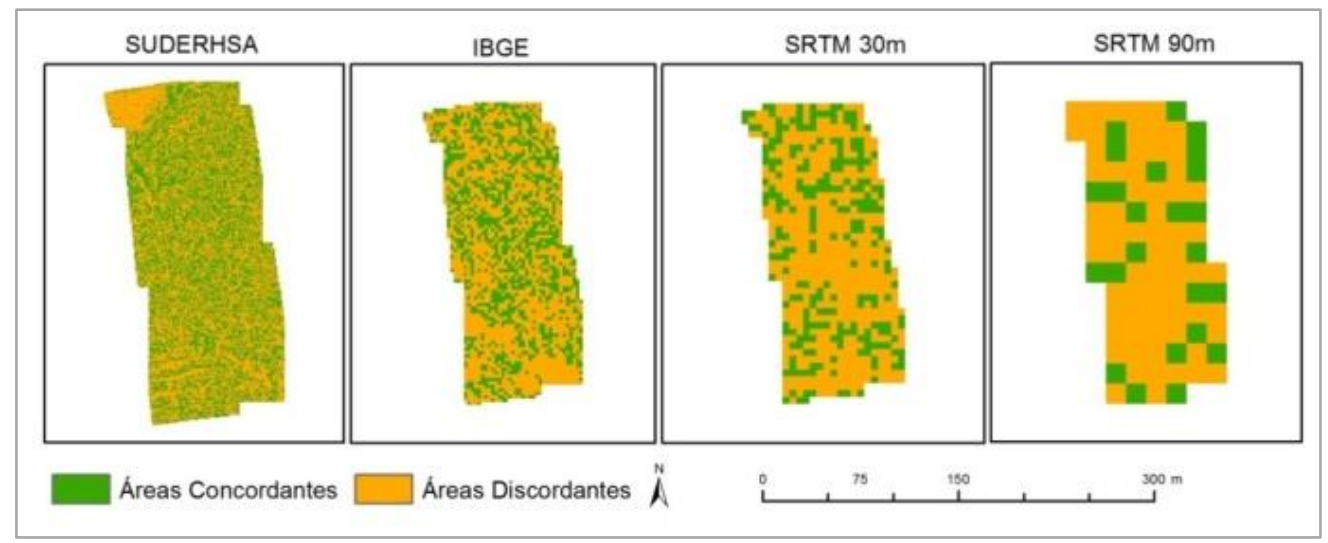

Figura 13 - Áreas concordantes e discordantes do atributo perfil de curvatura.

Apesar dos baixos índices de correlação entre as bases de dados, a avaliação visual revelou áreas homogêneas que associam padrões de relevo conhecidos da área de estudo, representados em mais de uma base de dados, como os observados na figura 5,8 e 9.

Isso enfatiza a necessidade de conhecimento das consequências em relação à escolha do modelo, em relação a sua forma de obtenção e resolução, sobre os parâmetros a serem obtidos. Embora as feições caracterizadas apresentem problemas, à utilização destes recursos é relevante em função da possibilidade de utilização em mapeamentos preliminares de menor custo servindo como subsídio a estudos científicos e técnicos.

\section{CONSIDERAÇÕES FINAIS}

Com base nos dados apresentados conclui-se que a utilização de diferentes modelos para a obtenção de atributos topográficos irá afetar de forma considerável os valores obtidos. Também é possível afirmar que a resolução dos modelos terá influência direta sobre os parâmetros morfométricos derivados.

Os valores de diferenças advêm da mensuração da variação espacial em função da utilização de matrizes de vizinhança e, portanto, o refinamento da quantidade de informações afeta a variação local dos índices obtidos em cada atributo, pois interfere nos resultados da aplicação de funções polinomiais tornando as representações um desafio.

A declividade apresentou para a área de estudo tendência de concentração de pixels na classe de declividade de 8 a 20\%, na medida em que se empregam modelos de menor resolução.
As curvaturas apresentaram valores próximos a zero nos modelos de menor resolução, gerando grandes agrupamentos de dados, e valores divergentes em modelos de maior resolução.

As variações apresentadas foram resultados do tamanho de pixel utilizado e da escala da base de dados. Esta é a dificuldade encontrada para utilizar objetivamente o potencial de aplicação e utilização dos dados geomorfométricos. As características observadas dependem da escala, mas esta não é uma limitação para todos os estudos. O resultado do trabalho depende largamente do domínio da aplicação e dos propósitos do estudo.

A pesquisa evidenciou a necessidade de se estabelecer critérios para a escolha de um MDE, uma vez que os modelos analisados apresentam divergências, o que torna os atributos suscetíveis à escolha do modelo e a resolução espacial do mesmo.

\section{REFERÊNCIAS}

BISPO, P. C.; VALERIANO, M. M.; KUPLICH, T. M. Variáveis geomorfométricas locais e sua relação com a vegetação da região do interflúvio Madeira-Purus (AM-RO). Acta Amazonica, v. 39, n. 01, p-81-90, 2009.

BUARQUE, D. C., FAN, F. M., PAZ, A., \& COLLISCHONN, W. Comparação de métodos para definir direções de escoamento a partir de modelos digitais de elevação. Revista Brasileira de Recursos Hídricos, n 14, p.91-103, 2009.

CÁTEN, A.; DALMOLIN, R. S. D.; RUIZ, L. F. C.; SEBEM, E.; PEREIRA, R. S. Pedometria aplicada à predição de classes de solos utilizando de 


\section{ANÁLISE DA INFLUÊNCIA DA ESCALA NA OBTENÇÃO DOS ATRIBUTOS TOPOGRÁFICOS DERIVADOS DE MDE}

regressões logísticas múltiplas. In: XIV SIMPÓSIO BRASILEIRO DE SENSORIAMENTO REMOTO, 2009, Natal. Anais Natal: INPE, p. 25-30, 2009.

CAMBOIM, S.P.; SLUTTER, C.R.; MENDONÇA, A.; JORGE, M.T.E. Mapeamento Sistemático: a base para as infraestruturas nacionais de dados espaciais. In: II SIMPÓSIO BRASILEIRO DE CIÊNCIAS GEODÉSICAS E TECNOLOGIAS DA GEOINFORMAÇÃO. Recife/PE, 8-11 de setembro de 2008 .

CENTENO, J. A. S. Sensoriamento Remoto e Processamento de Imagens Digitais. Departamento de Geomática da Universidade Federal do Paraná : Curitiba, 2003. 210p.

CHAGAS, C. S., Mapeamento digital de solos por correlação ambiental e redes neurais em uma bacia hidrográfica no domínio de mar de morros. Tese (Doutorado). Viçosa, 2006, Universidade Federal de Viçosa.

CHAGAS, C. S., FERNANDES FILHO, E. I., ROCHA, M. F., CARVALHO JÚNIOR, W. D., SOUZA NETO, N. C. Avaliação de modelos digitais de elevação para aplicação em um mapeamento digital de solos. Revista Brasileira de Engenharia Agrícola e Ambiental, v. 14, n. 2, p. 218-226, 2010.

EMBRAPA - EMPRESA BRASILEIRA DE PESQUISA AGROPECUÁRIA Sistema brasileiro de classificação de solos. Brasília, Serviço de Produção de Informação, 1999.

ESRI. ArcGis. versão 10.1. Environmental Systems Research Institute, 2012.

EVANS, I.S. General geomorphometry, derivatives of altitude and descriptive statistics. In SPATIAL ANALYSIS IN GEOMORPHOLOGY. London, Harper \&Row, 1972. pp. 17-90

FERREIRA, W. P. M., VALLADARES, G. S., \& HOTT, M. C. Estimativa da Temperatura Média Mensal do Ar para os Estados de Minas Gerais e do Pará, utilizando-se Modelos Digitais de Elevação. Engenharia na Agricultura, Viçosa, MG, v.14, n.4, 293-303, 2006.

FLORINSKY, I. V., EILERS, R. G., MANNING, G.R., FULLER, L.G. Prediction of soil properties by digital terrain modelling. Environmental Modelling \& Software, n 17,p 295-311, 2002.

GOMES, R.; GUIMARÃES, R; CARVALHO, O; FERNANDES, N; AMARAL, E. Combining Spatial Models for Shallow Landslides and Debris-Flows
Prediction. Remote Sensing, v. 5, p. 2219-2237, 2013.

GROHMANN, C.H. Introdução à Análise Digital de Terreno com GRASS-GIS. Instituto de Geociências. São Paulo: USP, 2007.

GROHMANN, C.H.; RICCOMINI, C.; STEINER, S.S. Aplicações dos Modelos de Elevação SRTM em Geomorfologia. Revista Geografia Acadêmica, v.2, n.2, p. 73-83, 2008.

GUIMARÃES, R. F.; MONTGOMERY, D.R; GREENBERG, H.; FERNANDES, N; GOMES, R. A.T; CARVALHO JÚNIOR, O,A. Parameterization of Soil Properties for a Model of Topographic Controls on Shallow Landsliding: Application to Rio de Janeiro. Engineering Geology, Holanda, v. 69, p. 99-108, 2003.

GUTELIUS, B.: Engineering Applications of Airborne Scanning Lasers: Report from the Field. Photogrammetric Engineering and Remote Sensing v.64 n64, pp.246-253, 1998.

HENGL, T. Finding the right pixel size. Computers \& Geosciences, v. 32, n. 9, p. 1283-1298, 2006.

HORN, B. K. P. Hill shading and the reflectance map. Proceedings of the IEEE, n. 69, v.01, p. 1447, 1981.

HUTCHINSON, M. F. Calculation of hydrologically sound digital elevation models. Paper presented at Third International Symposium on Spatial Data Handling at Sydney, Australia, 1988.

IBGE. Instituto Brasileiro de Geografia e Estatística, 1992. Mapeamento Topográfico Sistemático, Folha MI2842-3 (Escala 1:50.000).

IBGE. Instituto Brasileiro de Geografia e Estatística. Manual Técnico de Pedologia, 2007.

IPPOLITI, G. A.; COSTA, L.M.; SCHAEFER, C. E .G. R.; FERNANDES FILHO, E. I.;GAGGERO, M. R.; SOUZA, E. Análise digital do terreno: ferramenta na identificação de pedoformas em microbacia na região de "Mar de Morros" (MG). Revista Brasileira de Ciência do Solo, v.29, p.269-276, 2005.

JACOBI, L. F.; SOUZA, A. M.; PEREIRA, J.E. Gráfico de controle de regressão aplicado na monitoração de processos. Revista Produção, v. 12, n. 1, p. 46-59, 2002.

JENESS, J. DEM Surface Tools. Jenness Enterprises: Flagstaff, USA, 2012. Disponível em: https://goo.gl/TTWPF1 


\section{ANÁLISE DA INFLUÊNCIA DA ESCALA NA OBTENÇÃO DOS ATRIBUTOS TOPOGRÁFICOS DERIVADOS DE MDE}

JONES, K.H. A comparison of algorithms used to compute hill slope as a property of the DEM. Computers\& Geosciences, v. 24, n. 4, p. 315-323, 1998.

KIENZLE, S. The effect of DEM raster resolution on first order, second order and compound terrain derivatives .Transactions. In: GIS, v. 8, n. 1, p. 83-111, 2004.

Li, Z.; ZHU, Q.; GOLD, C..Digital terrain modeling: principles and methodology. CRC, 2010.

MAACK, R..Geografia física do Estado do Paraná. Banco de desenvolvimento do Paraná, 1968.

MINEROPAR. Atlas geológico do Estado do Paraná. Minerais do Paraná; Curitiba, 2001.

MINEROPAR. Atlas geomorfológico do Estado do Paraná. Escala1:250.000 modelos reduzidos base , 1:500.00. Minerais do Paraná; Universidade Federal do Paraná. Curitiba, 2006.

MIRANDA, J.I. Fundamentos de sistemas de informações geográficas. Brasília: Embrapa Informação Tecnológica, 2005. 425p.

MONTGOMERY, D. R. Predicting landscape-scale erosion using digital elevation models. Comptes Rendus Geoscience. Paris, v. 335, p. 1121-1130, 2003.

MOORE, I. D.; GESSLER, G.A.; PETERSON, G.A. Soil attribute prediction using terrain analysis.Soil Science Society of America Journal, v.57, p.443452, 1993.

NASA - NATIONAL AERONAUTICS AND SPACE ADMINISTRATION. Digital topographic, Shutte Radar Topography Mission.Escala 1:100.000, 2000.

NOWATZKI, A. Utilização de atributos topográficos no mapeamento preliminar de solos da Bacia Hidrográfica do Rio Pequeno (Antonina/PR). Curitiba, 2013 (Dissertação de Mestrado - Universidade Federal do Paraná).

PENNOCK, D. J.; ZEBARTH, B. J.; JONG, E. Landform classification and soil distribution in hummocky terrain, Saskatchewan, Canada. Geoderma, v. 40, n. 3, p. 297-315, 1987.

PENNOCK, D. J. Terrain attributes, landform segmentation, and soil redistribution. Soil and Tillage Research,v. 69 (1-2), p. 15-26, 2003.

PINHEIRO, H. S. K. BARBOSA, A. M. DOS ANJOS, L. H. C., DE CARVALHO JÚNIOR, W., DA SILVA CHAGAS, C. Avaliação de diferentes fontes de dados na obtenção do modelo digital de elevação para mapeamento digital dos solos da bacia hidrográfica do Rio Guapi-Macacu, RJ. Simpósio Brasileiro de Sensoriamento Remoto, 15 (SBSR), p. 9136-9143, 2011.

PEREIRA, J. G.; PEREIRA, L. E.. Uso de imagens CBERS 2B e dados SRTM no mapeamento de áreas de vulnerabilidade ambiental na cidade de Corumbá-MS.Simpósio Brasileiro de Sensoriamento Remoto, 15 (SBSR), p. 2628-2635, 2011.

PIKE, R.J. A bibliography of terrain modeling (geomorphometry), the quantitative representation of topography.USGS, p 02-465, 1995.

PIKE, A. C., MÜELLER, T. G., MIJATOVIC, B., KOOSTRA, B. K., POULETTE, M. M., PREWITT, R. M., SHEARER, S. A. Topographic Indices: Impact of Data Source. Soil Science, №171, p 800-809, 2006.

PINHEIRO, H. S. K., DA SILVA CHAGAS, C., DE CARVALHO JÚNIOR, W., DOS ANJOS, L. H. C. Modelos de elevação para obtenção de atributos topográficos utilizados em mapeamento digital de solos. Pesquisa Agropecuária Brasileira, Brasília, v. 47, n. 9, p. 1384-1394, Set/2012.

RAAFLAUB, L.D.; COLLINS, M.J. The effect of error in gridded digital elevation models on the estimation of topographic parameters. Environmental Modelling\& Software, v. 21, n. 5, p. 710-732, 2006.

RIBEIRO, C. A. A. S., SOARES, V. P., OLIVEIRA, A. M. S., \& GLERIANI, J. M. O desafio da delimitação de áreas de preservação permanente. Revista Árvore, no 29, p 203-212, 2005.

SALAMUNI, E.; EBERT, H. D.; HASUI, Y. Morfotectônica da bacia sedimentar de Curitiba. Brazilian Journal of Geology, v. 34, n. 4, p. 469478, 2008.

SHARY,P.A.; SHARAYA, L.S.; MITUSOV, A.V. Fundamental quantitative methods of land surface analysis. Geoderma, v. 107, n. 1, p. 1-32, 2002.

SAMPAIO, T.V.M.; AUGUSTIN, C.H.R.R. Índice de concentração da rugosidade: uma nova proposta metodológica para o mapeamento e quantificação da dissecação do relevo como subsídio a cartografia geomorfológica. Revista Brasileira de Geomorfologia, v. 15, n. 1, 2014. 


\section{ANÁLISE DA INFLUÊNCIA DA ESCALA NA OBTENÇÃO DOS ATRIBUTOS TOPOGRÁFICOS DERIVADOS DE MDE}

SCHMIDT, J. ANDREW,R. Multi-scale landform characterization. Area 37, p.341-350, 2005.

SCHMIDT, J.; DIKAU, R. Extracting geomorphometric attributes and objects from digital elevation models semantics, methods, future needs. In: DIKAU, R.; SAURER, H. (Eds.), GIS for Earth Surface Systems. Analysis and Modelling of the Natural Environment. Schweizbart'sche erlagsbuchhandlung, pp. 153-173, 1999.

SHARY, P.A., SHARAYA, L.S., MITUSOV, A.V. Fundamental quantitative methods of land surface analysis. Geoderma, v. 107 (1-2), p. 1-32, 2002.

SILVEIRA, C.T. Análise digital do relevo na predição de unidades preliminares de mapeamento de solos. Tese de Doutorado. Departamento de Geografia, UFPR, 2010.

SILVEIRA, C.T., FIORI, A. P., FERREIRA, A. M., FELIPE, R. D. S., KEPEL FILHO, J. L., FOLADOR, R. M., COSTA, L. C.. Análise do fator de segurança da estabilidade das vertentes na bacia do rio Jacareí, Serra do Mar Paranaense. Revista Brasileira de Geomorfologia, v. 13, n. 3, 2012a

SILVEIRA, R.M.P; SILVEIRA, C.T.; OKA-FIORI, C. Análise digital do relevo empregada no mapeamento de unidades geomorfológicas. Revista Geografar, v. 7, n. 2, 2012b.

SILVEIRA, C.T. OKA-FIORI, C.; SANTOS, L. J. C; SIRTOLI, A. E.; SILVA, C. R. Pedometria apoiada em atributos topográficos com operações de tabulação cruzada por álgebra de mapas. Revista Brasileira de Geomorfologia,v. 13, n. 02, p. 12537, 2012c.

SILVEIRA, C.T. OKA-FIORI, C.; SANTOS, L. J. C; SIRTOLI, A. E.; SILVA, C. R.; BOTELHO, M. F. Soil prediction using artificial neural networks and topographic attributes. Revista Geoderma,v. 195196, p. 165-172, 2013A.

SILVEIRA, C.T.; FIORI, A.P.; FERREIRA, A.M.; GÓIS, J.R.; MIO, G.; SILVEIRA, R.M.P.; MASSULINI, N.E.B.; LEONARDI, T.M.H.; Emprego de atributos topográficos no mapeamento da susceptibilidade a processos geoambientais na bacia do Rio Jacarei/Paraná. Revista Sociedade e Natureza, Uberlândia, vol. 25, n. 3, p. 623-639, 2013b.

SILVEIRA, C. T., FIORI, A.P., SCHILIPACK, P., DIAS, S.M. Mapeamento Preliminar da Suscetibilidade Natural a Movimentos de Massa da Serra do Mar Paranaense Apoiado na Análise Digital do Relevo.
Revista Brasileira de Geomorfologia, v. 15, n. 1, 2014.

SIQUEIRA, L. G. P., Controle estatístico do processo, São Paulo:Pioneira, 1997. 130p.

SIRTOLI, A. E.; SILVEIRA, C. T.; MONTOVANI, L. E.; SIRTOLI, A. R. A.; OKA-FIORI, C. Atributos do relevo derivados de modelo digital de elevação e suas relações com solos. Scientia agraria, v.9, n.3, p.317-329, 2008.

SOPCHAKI, C.H; Influência do $\mathrm{N}$ amostral e das características do relevo na qualidade de modelos digitais do terreno. Tese (Doutorado). Curitiba, 2016, UFPR.

STABACH, G.P. Base Laser Scan. Escala 1:1.000. In: Emprego de MDE e MDT obtido por levantamento aéreo com sistema laser scanner na obtenção de elementos superficiais e atributos topográficos. Monografia de Graduação. Departamento de Geografia, UFPR, 2013.

SUDERHSA - Superintendêcnia de desenvolvimento de recursos hídricos e saneamento. Cartas topográficas. Escala 1:10.000, 2000.

TANG, G. YANG W, QIN H. . An Application of Using GIS Technology to Return Hillside Cultivated Land for Forestry and Grassland in Loess Plateau Region. Bulletin of Soil and Land Water Conservation, n22, p 46-50, 2002.

TEZA, C.T.V; BAPTISTA, G.M.M. Identificação do fenômeno ilhas urbanas de calor por meio de dados ASTER-KineticTemperature (III): metrópoles brasileiras. Simpósio Brasileiro de Sensoriamento Remoto, 12 (SBSR), p. 3911-3918, 2005.

THOMPSON, J.A.; BELL, J.C.; BUTLER, C.A. Digital elevation model resolution: effects on terrain attribute calculation and quantitative soillandscape modeling. Geoderma, v. 100, n. 1, p. 67-89, 2001.

TOBLER, W, R. Analytical cartography. The American Cartographer. P. 21-3, 1976.

VALERIANO, M. M. Dados topográficos. In: FLORENZANO, T. G Geomorfologia: conceitos e tecnologias atuais. São Paulo: Oficina de textos, 2008. p.72-104.

VALERIANO, M. M. Modelo digital de elevação com dados SRTM disponíveis para a América do 


\section{ANÁLISE DA INFLUÊNCIA DA ESCALA NA OBTENÇÃO DOS ATRIBUTOS TOPOGRÁFICOS DERIVADOS DE MDE}

Sul . Instituto Nacional de Pesquisas Espaciais. São José dos Campos, 2004. Disponível em:

http://150.163.34.249/col/sid.inpe.br/sergio/200 4/06.30.10.57/doc/publicacao.pdf

VALERIANO, M. M.; ROSSETTI, D. F. Topodata: Brazilian full coverage refinement of SRTM data. Applied Geography (Sevenoaks), v. 32, p. 300309, 2011.

WARREN, S. D., HOHMANN, M. G., AUERSWALD, K., MITASOVA, H. An evaluation of methods to determine slope using digital elevation data.Catena,.v. 58, n. 3, p. 215-233, 2004.

WILSON, J. P.; GALLANT, J. C. Digital terrain analysis, chapter 1. In: Wilson, J. P.; Gallant, J. C. (eds.). Terrain analysis: principles and applications. New York: John Wiley \& Sons, p.127, 2000.
WOOD, J. Visualizing Geomorphometry: Lessons from Information Visualization. Proceedings of Geomorphometry, Zurich, Switzerland, p. 9-12, 2009.

ZEVENBERGEN, L. W.; THORNE, C.R. Quantitative analysis of land surface topography. Earth surface processes and landforms, v. 12, n. 1, p. 47-56, 1987.

ZHANG, W.; MONTGOMERY, D.R. Digital elevation model grid size, landscape representation, and hydrologic simulations. Water resources research, v. 30, n. 4, p. 1019 1028, 1994.

ZHOU, Q.; LIU, X.. Analysis of errors of derived slope and aspect related to DEM data properties.Computers\& Geosciences, v. 30, n. 4, p. 369-378, 2004. 